\title{
Stable birational invariants and the Lüroth problem
}

\author{
Claire Voisin
}

\begin{abstract}
We describe recent progress on rationality and stable rationality questions. We discuss the cohomological or Chow decomposition of the diagonal, a very strong stably birationally invariant property which controls many of the previously defined stable birational invariants. On the other hand, it behaves very well under specialization and desingularization of mild singularities.
\end{abstract}

\section{Introduction}

The subject of this paper is the generalized Lüroth problem which asks for criteria distinguishing unirational varieties from rational or stably rational varieties. Here the definitions are the following:

Definition 1.1. A smooth projective variety $X$ over a field $K$ is unirational if there exist an integer $N$ and a dominant rational map $\Phi: \mathbb{P}_{K}^{N} \rightarrow X$.

Note that one can always (at least if $K$ is infinite) reduce to the case $N=n=\operatorname{dim} X$ by restricting $\Phi$ to a general linear subspace $\mathbb{P}_{K}^{n} \subset \mathbb{P}_{K}^{N}$.

Definition 1.2. A smooth projective variety $X$ over a field $K$ is rational if there exists a birational map $\mathbb{P}_{K}^{n} \rightarrow X . X$ is stably rational if $X \times \mathbb{P}^{r}$ is rational for some integer $r$.

Of course, all these notions can be reformulated using only the function field $K(X)$ of $X$, so that the smoothness or projectivity of $X$ is not important here. However, it is very important in practice to work with smooth projective models. To start with, we have the following basic lemma.

Lemma 1.3. A rational map $\phi: X \rightarrow Y$ between projective varieties $X$ and $Y$ with $X$ smooth is well-defined away from a closed algebraic subset of $X$ of codimension $\geq 2$.

We also have in characteristic 0 the Hironaka theorem on resolution of indeterminacies: 
TheOREm 1.4. Let $\phi: X \rightarrow Y$ be a rational map between projective varieties. Then there exists a smooth projective variety $X^{\prime}$ with a morphism $\tau: X^{\prime} \rightarrow X$ which is the composition of a finite number of blow-ups along smooth centers, such that $\phi \circ \tau$ is a morphism $\phi^{\prime}: X^{\prime} \rightarrow Y$.

In dimension 1 and 2 and over an algebraically closed field of characteristic 0 , unirationality implies rationality (hence a fortiori stable rationality). In the surface case, this follows from the Castenuovo characterization of smooth projective rational surfaces as those smooth projective surfaces $X$ satisfying $q(X)=p_{2}(X)=0$ (see [5]).

Starting from dimension 3, the answer to the Lüroth problem asking whether unirational varieties are rational is negative. Three methods have been developed in the 70's to solve this problem, namely the ClemensGriffiths method [10], the Iskovskikh-Manin method [22], and the ArtinMumford method [2]. Among them, only the Artin-Mumford method solves the stable Lüroth problem, exhibiting unirational threefolds which are not stably rational. The invariant used by Artin-Mumford is the Brauer group and it is a topological invariant for rationally connected varieties. ColliotThélène and Ojanguren [11] described the higher degree generalization of this invariant, which takes the form of unramified cohomology with torsion coefficients. The first next invariant is the group $H_{n r}^{3}(X, \mathbb{Q} / \mathbb{Z})$ which was reinterpreted in $[\mathbf{1 3}]$ as the group $\operatorname{Hdg}^{4}(X, \mathbb{Z}) / H^{4}(X, \mathbb{Z})_{\text {alg }}$, at least when $X$ is rationally connected smooth projective defined over $\mathbb{C}$. It was proved in [39] that this group is trivial if $X$ is rationally connected of dimension 3, and in fact the Colliot-Thélène-Ojanguren examples of unirational varieties for which this group is nontrivial are known to exist only starting from dimension 6 (it is likely that they exist starting from dimension 4).

In [38] and [36], we studied the notion of decomposition of the diagonal, which in the Chow-theoretic setting is equivalent to the universal triviality of the $\mathrm{CH}_{0}$ group, as beautifully explained in [4]. This led us to the discovery of many new obstructions to the stable rationality of unirational threefolds. In particular, we proved

THEOREM 1.5. [36] The very general quartic double solid is not stably rational.

Using a beautiful combination of this method as improved by ColliotThélène and Pirutka [12] with the Kollár degeneration [24], Totaro proved the following:

Theorem 1.6. [35] A very general hypersurface of degree $\geq 2\left\ulcorner\left(\frac{n+2}{3}\right)\right\urcorner$ in $\mathbb{P}^{n}, n \geq 3$, is not stably rational.

The goal of this paper is to survey these recent developments, and some further related results in the case of cubic hypersurfaces, whose (stable) irrationality remains one of the most challenging problems in the field. 
Thanks. I thank Ludmil Katzarkov for his invitation to lecture on these topics in Miami, and Shing-Tung Yau for giving me the opportunity to present these new developments in this volume.

\section{Stable birational invariants}

We will discuss in this section quantities or properties of a smooth complex projective variety which are invariant under stable birational equivalence. Here we have in mind the following definition:

Definition 2.1. Two smooth projective varieties $X$ and $Y$ are stably birational if $X \times \mathbb{P}^{r} \stackrel{\text { birat }}{\cong} Y \times \mathbb{P}^{s}$ for some integers $r, s$.

In particular, $X$ is stably rational if and only if it is stably birationally equivalent to a point. We will first discuss stable birational invariants which come from topology or complex differential geometry. As we will see, very few of them can be nontrivial if the variety is unirational. We will then introduce more refined tools such as Chow groups and Hodge structures.

\subsection{Topology.}

2.1.1. Holomorphic forms. If $X$ is smooth projective over $K$, we can consider the spaces $H^{0}\left(X, \Omega_{X / K}^{\otimes k}\right)$, where $\Omega_{X / K}$ is the sheaf of Kähler differentials. If $K=\mathbb{C}$, by GAGA [32], this space identifies to the space $H^{0}\left(X^{a n}, \Omega_{X^{a n}}^{\otimes k}\right)$, where $X^{a n}$ is the complex manifold associated to $X$. We have the following lemma:

Lemma 2.2. For any $k \geq 0$, the space $H^{0}\left(X, \Omega_{X / K}^{\otimes k}\right)$ is a stable birational invariant.

Proof. We have to check first that this space does not change if $X$ is replaced by $X \times \mathbb{P}_{K}^{r}$. We have

$$
\Omega_{X \times \mathbb{P}^{r} / K}=p_{1}^{*} \Omega_{X / K} \oplus p_{2}^{*} \Omega_{\mathbb{P}_{K}^{r} / K},
$$

where the $p_{i}$ 's are the projections from $X \times \mathbb{P}^{r}$ to its factors. Thus $\Omega_{X \times \mathbb{P}_{K}^{r} / K}^{\otimes k}=\oplus_{p+q=k} p_{1}^{*} \Omega_{X / K}^{\otimes p} \otimes p_{2}^{*} \Omega_{\mathbb{P}_{K}^{r} / K}^{\otimes q}$ and

$$
H^{0}\left(X \times \mathbb{P}_{K}^{r}, \Omega_{X \times \mathbb{P}_{K}^{r} / K}^{\otimes k}\right)=\oplus_{p+q=k} H^{0}\left(X, \Omega_{X / K}^{\otimes p}\right) \otimes H^{0}\left(\mathbb{P}_{K}^{r}, \Omega_{\mathbb{P}_{K}^{r} / K}^{\otimes q}\right) .
$$

Then the result follows from $H^{0}\left(\mathbb{P}_{K}^{r}, \Omega_{\mathbb{P}_{K}^{r} / K}^{\otimes q}\right)=0$ for $q>0$ (and this space is obviously equal to $K$ for $q=0$ ). We next have to show that this space is invariant under birational equivalence. Let $\phi: X \rightarrow Y$ be a birational map. By Lemma 1.3, there exists a Zariski open set $U \subset X$ such that $\operatorname{codim} X \backslash U \geq 2$ and $\phi$ is well defined on $U$. We can thus define (because $k \geq 0)$

$$
\phi^{*}: H^{0}\left(Y, \Omega_{Y / K}^{\otimes k}\right) \rightarrow H^{0}\left(U, \Omega_{U / K}^{\otimes k}\right) .
$$

This morphism is injective because $\phi$ is generically of maximal rank. As $X$ is normal or by Hartogs in the complex setting, we have $H^{0}\left(U, \Omega_{U / K}^{\otimes k}\right)=$ 
$H^{0}\left(X, \Omega_{X / K}^{\otimes k}\right)$. We thus constructed an injective morphism $\phi^{*}: H^{0}\left(Y, \Omega_{Y / K}^{\otimes k}\right)$

$\rightarrow H^{0}\left(X, \Omega_{X / K}^{\otimes k}\right)$, which admits as inverse $\left(\phi^{-1}\right)^{*}$.

This fact is of course very important for birational geometry, but it is not useful for the Lüroth problem, by the following lemma:

LEMMA 2.3. Let $X$ be a rationally connected variety over a field $K$ of characteristic 0 . Then $H^{0}\left(X, \Omega_{X / K}^{\otimes k}\right)=0$ for $k>0$.

REMARK 2.4. This lemma remains true in characteristic different from 0 if "rationally connected" is replaced with "separably rationally connected".

Proof of Lemma 2.3. By definition, there exists a dominating rational map

$$
\Phi: Y \times \mathbb{P}_{K}^{1} \rightarrow X
$$

which maps $Y \times 0$ to $x \in X(K)$. As in the previous proof, the map $\Phi$ induces for any $k \geq 0$ an injective pull-back morphism $\Phi^{*}: H^{0}\left(X, \Omega_{X / K}^{\otimes k}\right) \rightarrow H^{0}(Y \times$ $\left.\mathbb{P}^{1}, \Omega_{Y \times \mathbb{P}_{K}^{1} / K}^{\otimes k}\right)$. We already saw that $H^{0}\left(Y \times \mathbb{P}^{1}, \Omega_{Y \times \mathbb{P}_{K}^{1} / K}^{\otimes k}\right) \cong H^{0}\left(Y, \Omega_{Y / K}^{\otimes k}\right)$, where the isomorphism is given by restriction to $Y \times 0$. On the other hand, we have for any $\alpha \in H^{0}\left(X, \Omega_{X / K}^{\otimes k}\right)$

$$
\left(\Phi^{*} \alpha\right)_{\mid Y \times 0}=\left(\Phi_{\mid Y \times 0}\right)^{*} \alpha=0
$$

for $k>0$ since $\Phi(Y \times 0)=\{x\}$.

REMARK 2.5. We used above the fact that $Y \times 0 \subset Y \times \mathbb{P}^{1}$ is a divisor, so that $\Phi$ is well defined generically along $Y \times 0$.

We have the following corollary:

Corollary 2.6. Let $X$ be a smooth rationally connected variety over $\mathbb{C}$. Then $H^{i}\left(X, \mathcal{O}_{X}\right)=0$ for $i>0$. This remains true over any field of characteristic 0 .

Proof. Indeed, we can use the Hodge symmetry that will be discussed in Section 2.2.3 and gives a $\mathbb{C}$-antilinear isomorphism between $H^{i}\left(X, \mathcal{O}_{X}\right)$ and $H^{0}\left(X, \Omega_{X}^{i}\right)$.

2.1.2. Fundamental group. The fundamental group $\pi_{1}\left(X^{a n}\right)$ of a smooth complex algebraic variety $X$ does not change when $X$ is replaced by $U=$ $X \backslash F$, where $F$ is a closed algebraic subset of codimension $\geq 2$. It follows that if $\phi: X \rightarrow Y$ is a rational map between smooth complex algebraic varieties, there is an induced morphism $\phi_{*}: \pi_{1}(X, x) \cong \pi_{1}(U, x) \rightarrow \pi_{1}(Y, \phi(x))$ where $x \in U$ and $U$ is the open set where $\phi$ is well-defined. When $\phi$ is birational, we can do the same thing for $\phi^{-1}$. We then conclude

LEMMA 2.7. The fundamental group of a smooth projective algebraic variety is a stable birational invariant. 
On the other hand, this invariant is not useful for the Lüroth problem, by the following result, which is due to Serre [31] in the unirational case.

LEMMA 2.8. Let $X$ be a smooth projective rationally connected complex variety. Then $\pi_{1}\left(X^{\text {an }}\right)$ is trivial.

Proof. First of all, $\pi_{1}\left(X^{a n}\right)$ is finite. This is proved as follows : there exists a dominating rational map

$$
\Phi: Y \times \mathbb{P}^{1} \rightarrow X
$$

which maps $Y \times 0$ to $x \in X$. We now use the fact that any surjective morphism $\psi: T \rightarrow X$ between complex algebraic varieties induces a morphism $\psi_{*}: \pi_{1}\left(T^{a n}\right) \rightarrow \pi_{1}\left(X^{a n}\right)$ whose image has finite index. We apply this to any birational model $T=\widehat{Y \times \mathbb{P}^{1}}$ on which $\Phi$ is a well defined morphism $\psi$. By Lemma 2.7, $T^{a n}$ has the same fundamental group as $(Y \times 0)^{a n}$, and thus we conclude that $\psi_{*}\left(\pi_{1}\left(T^{a n}\right)\right)=\Phi_{*}\left(\pi_{1}\left(Y^{a n} \times 0\right)\right)$. The later group is trivial since $\Phi_{\mid Y \times 0}$ is constant. This proves finiteness. The end of the argument is due to Serre. Let $u: \widetilde{X^{a n}} \rightarrow X^{a n}$ be the universal cover. The compact complex manifold $\widetilde{X^{a n}}$ is the analytisation of a smooth complex projective variety $\tilde{X}$. We have $\chi\left(\widetilde{X}, \mathcal{O}_{\tilde{X}}\right)=N \chi\left(X, \mathcal{O}_{X}\right)$, where $N=\operatorname{deg} u=\left|\pi_{1}\left(X^{a n}\right)\right|$. On the other hand, $\widetilde{X}$ is also rationally connected since $\mathbb{C P}^{1}$ is simply connected so any morphism from $\mathbb{P}^{1}$ to $X$ lifts to a morphism from $\mathbb{P}^{1}$ to $\widetilde{X}$. Thus $H^{i}\left(\widetilde{X}, \mathcal{O}_{\tilde{X}}\right)=0$ for $i>0$ by Lemma 2.6, and this implies that $\chi\left(\widetilde{X}, \mathcal{O}_{\tilde{X}}\right)=1$. Thus $N=1$ and $X=\tilde{X}$.

2.1.3. The Artin-Mumford invariant. In this section, we introduce a stable birational invariant which is topological (although it can be described in a purely algebraic way as part of the Brauer group) but can be nontrivial for some unirational varieties, as was discovered by Artin and Mumford [2]. The result is the following.

LEMmA 2.9. The group Tors $H_{B}^{3}(X, \mathbb{Z})$ is a stable birational invariant for smooth projective varieties. In particular, a stably rational variety has Tors $H_{B}^{3}(X, \mathbb{Z})=0$.

Here we denote $H_{B}^{i}(X, A)$ the $i$ th Betti cohomology of $X^{\text {an }}$, or of $X(\mathbb{C})$ equipped with the Euclidean topology, with coefficients in $A$. The group Tors $H_{B}^{3}(X, \mathbb{Z})$ is called the Artin-Mumford invariant of $X$.

Proof of Lemma 2.9. The fact that the group does not change when $X$ is replaced by $X \times \mathbb{P}^{r}$ follows from

$$
H_{B}^{3}\left(X \times \mathbb{P}^{r}, \mathbb{Z}\right)=H_{B}^{3}(X, \mathbb{Z}) \oplus H_{B}^{1}(X, \mathbb{Z}),
$$

where the $\operatorname{map} H_{B}^{1}(X, \mathbb{Z}) \rightarrow H_{B}^{3}\left(X \times \mathbb{P}^{r}, \mathbb{Z}\right)$ is given by $\alpha \mapsto p_{2}^{*} h \smile$ $p_{1}^{*} \alpha, h=c_{1}\left(\mathcal{O}_{\mathbb{P}^{r}}(1)\right)$. (Here the $p_{i}$ 's denote the projections from $X \times \mathbb{P}^{r}$ to its factors.) On the other hand, $H_{B}^{1}(X, \mathbb{Z})$ has no torsion (this follows from the consideration of the long exact sequence associated to the short 
exact sequence of sheaves $0 \rightarrow \mathbb{Z} \stackrel{n}{\rightarrow} \mathbb{Z} \rightarrow \mathbb{Z} / n \rightarrow 0$ on $\left.X^{a n}\right)$. It remains to prove birational invariance: if $\phi: Y \rightarrow X$ is birational, let

$$
\tau: \tilde{Y} \rightarrow Y, \tilde{\phi}: \tilde{Y} \rightarrow X
$$

be a desingularization of $\phi$. As $\tilde{\phi}$ has degree 1 , the morphism $\tilde{\phi}^{*}$ : $H_{B}^{3}(X, \mathbb{Z}) \rightarrow H_{B}^{3}(\widetilde{Y}, \mathbb{Z})$ is injective, because $\tilde{\phi}_{*} \circ \tilde{\phi}^{*}=I d$ on cohomology. On the other hand $\tau$ is a sequence of blow-ups along smooth centers and it follows from this that $\tau_{*}:$ Tors $H_{B}^{3}(\widetilde{Y}, \mathbb{Z}) \rightarrow$ Tors $H_{B}^{3}(Y, \mathbb{Z})$ is an isomorphism. Indeed, for a single blow-up along a smooth $Z \subset Y$, we have

$$
H_{B}^{3}(\tilde{Y}, \mathbb{Z})=H_{B}^{3}(Y, \mathbb{Z}) \oplus H_{B}^{1}(Z, \mathbb{Z})
$$

and thus Tors $H_{B}^{3}(\widetilde{Y}, \mathbb{Z})=\operatorname{Tors} H_{B}^{3}(Y, \mathbb{Z})$. Thus we conclude that $\tau_{*} \circ \tilde{\phi}^{*}$ injects Tors $H_{B}^{3}(X, \mathbb{Z})$ in Tors $H_{B}^{3}(Y, \mathbb{Z})$. To prove that

$$
\tau_{*} \circ \tilde{\phi}^{*}: \text { Tors } H_{B}^{3}(X, \mathbb{Z}) \rightarrow \text { Tors } H_{B}^{3}(Y, \mathbb{Z})
$$

is surjective, one can construct by a finite sequence of blow-ups a variety $\widetilde{X} \stackrel{\tau^{\prime}}{\rightarrow} X$ dominating $\tilde{Y}$, via a morphism $\psi$ desingularizing $\phi^{-1}$. Then $\tau^{\prime *}$ : Tors $H_{B}^{3}(X, \mathbb{Z}) \rightarrow$ Tors $H_{B}^{3}(\widetilde{X}, \mathbb{Z})$ is surjective and since $\psi_{*}$ is surjective, this implies as above that $\tilde{\phi}^{*}:$ Tors $H_{B}^{3}(X, \mathbb{Z}) \rightarrow$ Tors $H_{B}^{3}(\widetilde{Y}, \mathbb{Z})$ is surjective.

Artin and Mumford constructed in [2] unirational threefolds with nontrivial Artin-Mumford invariant, providing the first examples of unirational not stably rational threefolds. Their construction is as follows. We consider quartic double solids $X \rightarrow \mathbb{P}^{3}$ ramified along a quartic surface $S \subset \mathbb{P}^{3}$. Such a variety $X$ is defined as a hypersurface in the affine fibration $p: \mathbb{L}=$ Spec $\left(\operatorname{Sym} \mathcal{O}_{\mathbb{P}^{3}}(-2)\right) \rightarrow \mathbb{P}^{3}$ (the total space of the line bundle $\left.\mathcal{O}_{\mathbb{P}^{3}}(2)\right)$ by the following equation: $\mathbb{L}$ has a canonical nonzero section $u \in H^{0}\left(\mathbb{L}, p^{*} \mathcal{O}(2)\right)$ and $S$ is defined as the zero set of a section $f \in H^{0}\left(\mathbb{P}^{3}, \mathcal{O}_{\mathbb{P}^{3}}(4)\right)$. Then $X \subset \mathbb{L}$ is defined by the equation $u^{2}=p^{*} f$. Assume now that $S$ has an ordinary double point $P$. The equation $f$ can then be written as

$$
f=X_{0}^{2} q\left(X_{1}, X_{2}, X_{3}\right)+X_{0} t\left(X_{1}, X_{2}, X_{3}\right)+f_{4}\left(X_{1}, X_{2}, X_{3}\right),
$$

where $X_{1}, X_{2}, X_{3}$ vanish at $P$, and $\operatorname{deg} q=2, \operatorname{deg} f_{4}=4, \operatorname{deg} t=3$. Projecting from $P$, we see that $S$, or rather its blow-up at $P$, is a double cover of $\mathbb{P}^{2}$ ramified along the sextic curve $C$ with equation $t\left(X_{1}, X_{2}, X_{3}\right)^{2}-$ $4 q\left(X_{1}, X_{2}, X_{3}\right) f_{4}\left(X_{1}, X_{2}, X_{3}\right)$. The condition imposed by Artin and Mumford is the following: the curve $C$ is the union of two cubic curves meeting transversally. This imposes nine supplementary double points to the $K 3$ surface $S$, namely one for each node of $C$. Thus $S$, hence also $X$, has 10 ordinary double points. Let $\widetilde{X}$ be the blow-up of $X$ at its singular points.

TheOREM 2.10. [2] The variety $\widetilde{X}$ has some nontrivial 2-torsion in $H_{B}^{3}(\widetilde{X}, \mathbb{Z})$. In particular it is not stably rational.

On the other hand, it is easy to check that $\widetilde{X}$ is unirational. Indeed, choose a node of $X$, corresponding to a node $P$ of $S$. The lines in $\mathbb{P}^{3}$ passing 
through $P$ meet $S$ in three points, namely $P$ counted with multiplicity 2 and two other points $x, y$. The inverse image of such a line in $X$ is thus a curve whose normalization at $P$ is a double cover of $\mathbb{P}^{1}$ ramified at only 2 points, hence is a rational curve. This construction gives $\widetilde{X}$ the structure of a conic bundle over the $\mathbb{P}^{2}$ parameterizing lines in $\mathbb{P}^{3}$ through $P$. On the other hand, let $H \subset \mathbb{P}^{3}$ be a general plan. The inverse image $\Sigma$ of $H$ in $\widetilde{X}$ is a del Pezzo surface, hence is rational. The fibered product $Y:=\Sigma \times_{\mathbb{P}^{2}} \widetilde{X}$ is a conic bundle over $\Sigma$ which admits a section, hence is rational. As it dominates $\widetilde{X}$, the later is unirational.

\subsection{Further tools.}

2.2.1. Chow groups. Let $X$ be an algebraic variety over a field $K$. Let $\mathcal{Z}_{k}(X)$ be the free abelian group generated by the subvarieties (that is, irreducible closed algebraic subsets) $Z \subset X$ of dimension $k$. If $\phi: X \rightarrow Y$ is a proper morphism, one defines

$$
\phi_{*}: \mathcal{Z}_{k}(X) \rightarrow \mathcal{Z}_{k}(Y)
$$

on generators by $\phi_{*}(Z)=0$ if $\operatorname{dim} \phi(Z)<k$ and $\phi_{*}(Z)=(\operatorname{deg} Z / \phi(Z)) \phi(Z)$, where the integer $\operatorname{deg} Z / \phi(Z)$ is the degree of the field extension $K(\phi(Z)) \subset$ $K(Z)$. (If $K=\mathbb{C}$ and $\phi(Z)$ is smooth, this is also the topological degree of $\phi: Z^{a n} \rightarrow \phi(Z)^{a n}$.)

The Chow groups $\mathrm{CH}_{k}(X)$ are defined as the quotients $\mathcal{Z}_{k}(X) / \mathcal{Z}_{k}(X)_{\text {rat }}$ where the subgroup $\mathcal{Z}_{k}(X)_{\text {rat }} \subset \mathcal{Z}_{k}(X)$ is generated by the following cycles $n_{*}(\operatorname{div} f)$ said rationally equivalent to 0 : for any irreducible closed algebraic subset $W \subset X$ of dimension $k+1$, and any nonzero rational function $f \in K(W)^{*}$, letting $n: \widetilde{W} \rightarrow X$ be the normalization of $W$, we get $n_{*}(\operatorname{div} f) \in \mathcal{Z}_{k}(X)$.

If $X$ is smooth of dimension $n$, we will denote $\mathrm{CH}^{n-k}(X):=\mathrm{CH}_{k}(X)$. Chow groups (particularly on smooth projective varieties) have excellent functoriality properties:

a) If $\phi: X \rightarrow Y$ is a proper morphism, the map $\phi_{*}$ defined above on cycles factors through rational equivalence and provides $\phi_{*}: \mathrm{CH}_{k}(X) \rightarrow$ $\mathrm{CH}_{k}(Y)$.

b) If $X$ and $Y$ are smooth, and $\phi: X \rightarrow Y$ is any morphism, there is a pull-back morphism $\phi^{*}: \mathrm{CH}^{k}(Y) \rightarrow \mathrm{CH}^{k}(X)$. If $\phi$ is flat, $\phi^{*}$ is induced by the morphism $\phi^{*}: \mathcal{Z}^{k}(Y) \rightarrow \mathcal{Z}^{k}(X)$ which to $Z$ associates the cycle of $\phi^{-1}(Z)$. Here $\phi^{-1}(Z)$ has the right codimension by flatness but it is not irreducible or reduced in general, and its cycle is the sum of its irreducible components counted with adequate multiplicities, the "lengthes".

c) If $Y$ is smooth, there is an intersection product $\mathrm{CH}^{k}(Y) \otimes \mathrm{CH}^{l}(Y) \rightarrow$ $\mathrm{CH}^{k+l}(Y)$, which maps $Z \otimes Z^{\prime}$ to $i_{\Delta}^{*}\left(Z \times Z^{\prime}\right)$ where $i_{\Delta}$ is the diagonal inclusion of $Y$ in $Y \times Y$. If $X$ and $Y$ are smooth, and $\phi: X \rightarrow Y$ is any morphism, the pull-back map $\phi^{*}: \mathrm{CH}^{*}(Y) \rightarrow \mathrm{CH}^{*}(X)$ is compatible with the intersection product: $\phi^{*}\left(Z \cdot Z^{\prime}\right)=\phi^{*} Z \cdot \phi^{*} Z^{\prime}$. 
d) (Projection formula) If $\phi: X \rightarrow Y$ is a projective morphism between smooth varieties, one has for $Z \in \mathrm{CH}(X), Z^{\prime} \in \mathrm{CH}(Y), \phi_{*}\left(Z \cdot \phi^{*}\left(Z^{\prime}\right)\right)=$ $Z^{\prime} \cdot \phi_{*}(Z)$ in $\mathrm{CH}(Y)$.

Chow groups also have a very good property which makes them easier to handle than cohomology or homology, namely if $F \subset X$ is a closed algebraic subset and $U=X \backslash F$, then we have the localization exact sequence

$$
\mathrm{CH}_{i}(F) \rightarrow \mathrm{CH}_{i}(X) \rightarrow \mathrm{CH}_{i}(U) \rightarrow 0
$$

where the last map is restriction to $U$ (or intersection with $U$ ) and is a particular case of the flat pull-back mentioned in b) above, and the first map is the proper pushforward $i_{*}$ mentioned in a), where $i$ is the inclusion of $F$ in $X$.

2.2.2. Birational invariance of $\mathrm{CH}_{0}$. The group $\mathrm{CH}_{0}(X)$ is a very interesting invariant of a smooth projective variety. Over $\mathbb{C}$ and in the case of curves, it has been completely understood thanks to Abel's theorem (see Section 3.2.1). In higher dimension (and still over $\mathbb{C}$ ), it is conjectured that the group $\mathrm{CH}_{0}(X)$ is trivial (that is, equal to $\mathbb{Z}$ ) if $H^{0}\left(X, \Omega_{X}^{k}\right)=0$ for $k>0$. This is a famous conjecture due to Bloch.

Let us prove the following easy fact:

Lemma 2.11. The group $\mathrm{CH}_{0}(X)$ is a stable birational invariant of smooth projective varieties.

Proof. First of all, $\mathrm{CH}_{0}\left(\mathbb{P}_{K}^{r}\right)=\mathbb{Z}$ as follows from the localization exact sequence $(1)$ and $\mathrm{CH}_{0}\left(\mathbb{A}_{K}^{s}\right)=0$ for $s>0$. The same argument proves more generally that $\mathrm{CH}_{0}\left(X \times \mathbb{P}_{K}^{r}\right)=\mathrm{CH}_{0}(X)$, where the morphism $\mathrm{CH}_{0}(X) \rightarrow \mathrm{CH}_{0}\left(X \times \mathbb{P}_{K}^{r}\right)$ is $i_{x *}$ for any $x \in \mathbb{P}_{K}^{r}(K), i_{x}$ being the inclusion $X \cong X \times x \hookrightarrow \mathbb{P}_{K}^{r}$.

It remains to prove birational invariance. If $\phi: X \rightarrow Y$ is birational, then we have the graph $\Gamma_{\phi} \subset X \times Y$ which has a class $\Gamma \in \mathrm{CH}^{n}(X \times Y)$, $n=\operatorname{dim} X=\operatorname{dim} Y$. We can then construct

$$
\Gamma^{*}: \mathrm{CH}(Y) \rightarrow \mathrm{CH}(X), \quad \Gamma_{*}: \mathrm{CH}(X) \rightarrow \mathrm{CH}(Y)
$$

by the formula

$$
\Gamma^{*}(z):=p r_{1 *}\left(\Gamma \cdot p r_{2}^{*} z\right), \quad \Gamma_{*}\left(z^{\prime}\right):=p r_{2 *}\left(\Gamma \cdot p r_{1}^{*} z^{\prime}\right)
$$

for $z \in \mathrm{CH}(Y), z^{\prime} \in \mathrm{CH}(X)$. The composition of two correspondences

$$
Z \in \mathrm{CH}(X \times Y), Z^{\prime} \in \mathrm{CH}(Y \times W)
$$

where $X, Y, W$ are smooth and projective over $K$ is the correspondence $Z^{\prime} \circ Z \in \mathrm{CH}(X \times W)$ defined as

$$
Z^{\prime} \circ Z=p r_{X W *}\left(p r_{X Y}^{*} Z \cdot p r_{Y W}^{*} Z^{\prime}\right),
$$

where the $p r_{\bullet \bullet}$ 's are the projections from $X \times Y \times W$ to the product of two of its factors. One easily checks that

$$
\left(Z^{\prime} \circ Z\right)_{*}=Z_{*}^{\prime} \circ Z_{*}: \mathrm{CH}(X) \rightarrow \mathrm{CH}(W) .
$$


Using this formalism, one now proves that $\Gamma_{*} \circ \Gamma^{*}=I d$ on $\mathrm{CH}_{0}(Y)$ and $\Gamma^{*} \circ \Gamma_{*}=I d$ on $\mathrm{CH}_{0}(X)$. One uses for this the easy fact that the cycles $\Gamma \circ{ }^{t} \Gamma \in \mathrm{CH}(Y \times Y)$, resp. ${ }^{t} \Gamma \circ \Gamma \in \mathrm{CH}(X \times X)$, differ from $\Delta_{Y}\left(\operatorname{resp} . \Delta_{X}\right)$ by a cycle supported on $E \times E$, where $E$ is proper closed algebraic subset of $Y$ (resp. $X)$. (Here the cycle ${ }^{t} \Gamma \in \mathrm{CH}(Y \times X)$ is the image of the cycle $\Gamma$ by the obvious isomorphism $X \times Y \cong Y \times X$.) Indeed, writing

$$
\Gamma \circ{ }^{t} \Gamma=\Delta_{Y}+Z \in \mathrm{CH}^{n}(Y \times Y)
$$

with $Z$ supported on $E \times Y$ for some proper closed algebraic subset $E \subset Y$, we observe that $Z_{*}=0$ on $\mathrm{CH}_{0}(Y)$ so that (2) gives

$$
\Gamma_{*} \circ{ }^{t} \Gamma_{*}=\Gamma_{*} \circ \Gamma^{*}=\Delta_{Y *}=I d
$$

on $\mathrm{CH}_{0}(Y)$, and similarly for $X$.

We will also use later on the projective bundle and blow-up formulas, which give another proof of the birational invariance of $\mathrm{CH}_{0}$ assuming desingularization (hence in characteristic 0). First of all, we always have from the definitions $\mathrm{CH}^{1}(X)=\operatorname{Pic} X$. In particular, if $\pi: P \rightarrow X$ is a projective bundle, $P=\mathbb{P}(\mathcal{E})$, we have the line bundle $\mathcal{O}_{\mathbb{P}(\mathcal{E})}(1)$ on $P$ which provides an element $h \in \mathrm{CH}^{1}(P)$.

Theorem 2.12. [15, Theorem 3.3] One has

$$
\mathrm{CH}^{i}(P)=\oplus_{k=0}^{k=\operatorname{Inf}(r-1, i)} \mathrm{CH}^{i-k}(X),
$$

where the maps from the right to the left are given by $\alpha \mapsto h^{k} \cdot \pi^{*} \alpha$, and $r=\operatorname{rank} \mathcal{E}$.

Let now $X$ be smooth projective, and $Z \subset X$ be a smooth closed subvariety of codimension $r$. Let $\tau: \widetilde{X}_{Z} \rightarrow X$ be the blow-up of $X$ along $Z$. Let $E$ be the exceptional divisor of $\tau$ and denote by $i_{E}$ the inclusion of $E$ in $\tilde{X}_{Z}, \tau_{E}: E \rightarrow Z$ the restriction of $\tau$ to $E$. The morphism $\tau_{E}$ makes $E$ into a $\mathbb{P}^{r-1}$-bundle, which admits as $\mathcal{O}(1)$-bundle the line bundle $\mathcal{O}_{E}(-E)$ of class $\delta \in \mathrm{CH}^{1}(E)$.

Theorem 2.13. [15, Proposition 6.7] One has

$$
\mathrm{CH}^{i}\left(\widetilde{X}_{Z}\right)=\mathrm{CH}^{i}(X) \oplus_{k=0}^{k=\operatorname{Inf}(r-2, i)} \mathrm{CH}^{i-k-1}(Z),
$$

where the maps from the right to the left are given by

$$
\tau^{*}: \mathrm{CH}^{i}(X) \rightarrow \mathrm{CH}^{i}\left(\widetilde{X}_{Z}\right), i_{E *} \circ \delta^{k} \cdot \circ \tau_{E}^{*}: \mathrm{CH}^{i-k-1}(Z) \rightarrow \mathrm{CH}^{i}\left(\widetilde{X}_{Z}\right) .
$$

2.2.3. Hodge structures and cycle classes. Let $X$ be a smooth projective complex variety. We have $H_{B}^{i}(X, \mathbb{C})=H_{B}^{i}(X, \mathbb{Z}) \otimes \mathbb{C}$ and on the other hand, Hodge theory provides the Hodge decomposition

$$
H_{B}^{i}(X, \mathbb{C})=\oplus_{p+q=i} H^{p, q}(X),
$$

where $H^{p, q}(X)$ is defined as the set of de Rham cohomology classes of closed forms of type $(p, q)$ on $X^{a n}$. This definition makes obvious the Hodge symmetry property, which says that $\overline{H^{p, q}(X)}=H^{q, p}(X)$, since the complex 
conjugate of a closed form of type $(p, q)$ is a closed form of type $(q, p)$. These data endow $H_{B}^{i}(X, \mathbb{Z}) /$ torsion with an integral Hodge structure of weight $i$. We concentrate in this section on even degree cohomology.

Definition 2.14. An integral Hodge class of degree $2 k$ on $X$ (notation $\left.\operatorname{Hdg}^{2 k}(X, \mathbb{Z})\right)$ is an integral cohomology class $\alpha \in H_{B}^{2 k}(X, \mathbb{Z})$ whose image $\alpha_{\mathbb{C}}$ in $H_{B}^{2 k}(X, \mathbb{C})$ belongs to $H^{k, k}(X)$.

Let $Z \subset X$ be a closed irreducible subset of codimension $k$. Let $j: \widetilde{Z} \rightarrow X$ be a desingularization of $Z$. Then as a compact complex manifold, $\widetilde{Z}^{a n}$ has a fundamental homology class $[\widetilde{Z}]_{\text {fund }} \in H_{2 n-2 k}\left(\widetilde{Z}^{a n}, \mathbb{Z}\right)$ whose image in $H_{B}^{2 k}(X, \mathbb{Z})$ by the composite map

$$
\begin{aligned}
& H_{2 n-2 k}\left(\widetilde{Z}^{a n}, \mathbb{Z}\right) \stackrel{j_{*}}{\rightarrow} H_{2 n-2 k}\left(X^{a n}, \mathbb{Z}\right) \stackrel{\text { Poincaré duality }}{\cong} H^{2 k}\left(X^{a n}, \mathbb{Z}\right) \\
& \quad=H_{B}^{2 k}(X, \mathbb{Z}),
\end{aligned}
$$

where $n=\operatorname{dim} X$, is called the cycle class of $Z$.

More generally, if $Z=\sum_{i} n_{i} Z_{i}$, one defines $[Z] \in H_{B}^{2 k}(X, \mathbb{Z})$ as $\sum_{i} n_{i}\left[Z_{i}\right]$. It is easy to prove that the cycle class so defined on $\mathcal{Z}^{k}(X)$ factors through rational equivalence to give

$$
c l: \mathrm{CH}^{k}(X) \rightarrow H_{B}^{2 k}(X, \mathbb{Z}) .
$$

Lemma 2.15. The image of $c l$ is contained in $\operatorname{Hdg}^{2 k}(X, \mathbb{Z})$.

Proof. Indeed, a class $\alpha_{\mathbb{C}} \in H^{2 k}\left(X^{a n}, \mathbb{C}\right)$ belongs to $H^{k, k}(X)$ if and only if for any $\beta \in H^{p, q}(X), p+q=2 n-2 k$, with $(p, q) \neq(n-k, n-k)$, one has $\left\langle\alpha_{\mathbb{C}}, \beta\right\rangle_{X}$. In the case of $\alpha=[Z]$, we have

$$
\left\langle\alpha_{\mathbb{C}}, \beta\right\rangle_{X}=\int_{\widetilde{Z}^{a n}} j^{*} \beta,
$$

hence the vanishing follows from the vanishing of a form $j^{*} \beta$ on $\widetilde{Z}^{a n}$ when $\beta$ is of type $(p, q), p+q=2 n-2 k,(p, q) \neq(n-k, n-k)$.

\subsection{Stable birational invariants from cycle class.}

2.3.1. The Lefschetz $(1,1)$-theorem. The simplest example of the cycle class is the case of codimension 1 cycles. Assume $X$ is smooth and projective. Recall that $\mathrm{CH}^{1}(X)=\operatorname{Pic} X$. By GAGA [32], we have Pic $X=\operatorname{Pic} X^{a n}$ and the later group is isomorphic to $H^{1}\left(X^{a n}, \mathcal{O}_{X^{a n}}^{*}\right)$. We have the exponential exact sequence on $X^{\text {an }}$

$$
0 \rightarrow \mathbb{Z} \stackrel{2 \iota \pi}{\rightarrow} \mathcal{O}_{X^{a n}} \stackrel{\exp }{\longrightarrow} \mathcal{O}_{X^{a n}}^{*} \rightarrow 1,
$$

whose associated long exact sequence gives

$$
\operatorname{Pic} X^{a n} \stackrel{c_{1}}{\rightarrow} H^{2}\left(X^{a n}, \mathbb{Z}\right) \rightarrow H^{2}\left(X^{a n}, \mathcal{O}_{X^{a n}}\right)
$$

defining $c_{1}$. It is not hard to prove (see [43, Theorem 11.33]) that via the identification $\mathrm{CH}^{1}(X)=\operatorname{Pic} X^{a n}, c_{1}$ identifies to the cycle class map for 
divisors. The exact sequence (5) now gives us the Lefschetz theorem on $(1,1)$-classes:

TheOREM 2.16. The image of the cycle class map

$$
c l: \mathrm{CH}^{1}(X) \rightarrow H_{B}^{2}(X, \mathbb{Z})
$$

is the group of integral Hodge classes $\operatorname{Hdg}^{2}(X, \mathbb{Z}) \subset H_{B}^{2}(X, \mathbb{Z})$.

Proof. We only have to prove that $\operatorname{Hdg}^{2}(X, \mathbb{Z})$ identifies with $\operatorname{Ker}\left(H^{2}\left(X^{a n}, \mathbb{Z}\right) \rightarrow H^{2}\left(X, \mathcal{O}_{X^{a n}}\right)\right)$. Clearly, any cohomology class of type $(1,1)$, in particular any degree 2 Hodge class, maps to 0 in $H^{2}\left(X^{a n}, \mathcal{O}_{X^{a n}}\right)$. Conversely, if a degree 2 integral cohomology class $\alpha$ maps to 0 in $H^{2}\left(X^{a n}, \mathcal{O}_{X^{a n}}\right)$, then its $(0,2)$-component $\alpha^{0,2}$ vanishes in $H^{0,2}(X)=$ $H^{2}\left(X^{a n}, \mathcal{O}_{X^{a n}}\right)$. As $\alpha$ is real, $\alpha^{2,0}$ also vanishes by Hodge symmetry, hence $\alpha$ is of type $(1,1)$.

2.3.2. Integral Hodge classes of degree 4. We use now the Lefschetz theorem on $(1,1)$-classes to prove the following

Proposition 2.17. (See [33]) The group $Z^{4}(X):=\operatorname{Hdg}^{4}(X, \mathbb{Z}) /$ $H_{B}^{4}(X, \mathbb{Z})_{a l g}$ is a stable birational invariant of a smooth projective complex variety $X$.

Proof. We have

$$
\operatorname{Hdg}^{4}\left(X \times \mathbb{P}^{r}, \mathbb{Z}\right)=p r_{1}^{*} \operatorname{Hdg}^{4}(X, \mathbb{Z}) \oplus p r_{2}^{*}[h] \smile p r_{1}^{*} \operatorname{Hdg}^{2}(X, \mathbb{Z}),
$$

where $h=c_{1}\left(\mathcal{O}_{\mathbb{P}^{r}}(1)\right),[h]=c l(h)$ and the $p r_{i}$ are the obvious projections.

Similarly and in a compatible way with respect to the cycle class map, we have by Theorem 2.12

$$
\mathrm{CH}^{2}\left(X \times \mathbb{P}^{r}\right)=p r_{1}^{*} \mathrm{CH}^{2}(X) \oplus p r_{2}^{*} h \cdot p r_{1}^{*} \mathrm{CH}^{1}(X) .
$$

The equality $Z^{4}\left(X \times \mathbb{P}^{r}\right)=Z^{4}(X)$ then follows from the Lefschetz theorem on $(1,1)$-classes, which says that $\operatorname{cl}\left(\mathrm{CH}^{1}(X)\right)=\operatorname{Hdg}^{2}(X, \mathbb{Z})$.

Next assume $\phi: Y \rightarrow X$ is a birational map. As usual, let $\tilde{\phi}: Y^{\prime} \rightarrow X$ be a desingularization of $\phi$, where $Y^{\prime}$ is deduced from $Y$ by a sequence of blow-ups. Let $\alpha$ be a degree 4 integral Hodge class on $X$. Then $\alpha$ is algebraic if and only if $\tilde{\phi}^{*} \alpha$ is algebraic on $Y^{\prime}$, since $\tilde{\phi}_{*} \circ \tilde{\phi}^{*}=I d$ on $\operatorname{Hdg}^{4}(X, \mathbb{Z})$ and by compatibility of the cycle class map with the pull-back and push-forward maps. We claim now that

$$
Z^{4}\left(Y^{\prime}\right) \cong Z^{4}(Y)
$$

Indeed, we reduce to the case where $\tau: Y^{\prime} \rightarrow Y$ is the blow-up of a smooth subvariety $Z$. Then we have

$$
\operatorname{Hdg}^{4}\left(Y^{\prime}, \mathbb{Z}\right)=\tau^{*} \operatorname{Hdg}^{4}(Y, \mathbb{Z}) \oplus i_{*}\left(\tau_{E}^{*} \operatorname{Hdg}^{2}(Z, \mathbb{Z})\right) \oplus i_{*}\left([\delta] \smile \tau_{E}^{*} \operatorname{Hdg}^{0}(Z, \mathbb{Z})\right),
$$

where the last term appears only if $\operatorname{codim} Z \geq 3$. We have the similar formula for Chow groups (see Theorem 2.13)

$$
\mathrm{CH}^{2}\left(Y^{\prime}\right)=\tau^{*} \mathrm{CH}^{2}(Y) \oplus i_{*}\left(\tau_{E}^{*} \mathrm{CH}^{1}(Z)\right) \oplus i_{*}\left([\delta] \smile \tau_{E}^{*} C H^{0}(Z)\right),
$$


where the last term appears only if $\operatorname{codim} Z \geq 3$. We now use the Lefschetz $(1,1)$-theorem to conclude that $\operatorname{Hdg}^{2}(Z, \mathbb{Z})=\operatorname{cl}\left(\mathrm{CH}^{1}(Z)\right)$ and the obvious fact that $\operatorname{Hdg}^{0}(Z, \mathbb{Z})$ is generated by the class of $Z$, to conclude that $i_{*}\left(\tau_{E}^{*} \operatorname{Hdg}^{2}(Z, \mathbb{Z})\right) \oplus i_{*}\left([\delta] \smile \tau_{E}^{*} \operatorname{Hdg}^{0}(Z, \mathbb{Z})\right) \subset H_{B}^{4}\left(Y^{\prime}, \mathbb{Z}\right)_{\text {alg }}$ which proves the claim. The claim now concludes the proof of the proposition since we proved that

$$
\tilde{\phi}^{*}=\tau_{*} \circ \tilde{\phi}^{*}: Z^{4}(X) \rightarrow Z^{4}(Y)
$$

is injective, and we can prove surjectivity similarly by blowing-up $X$ rather than $Y$.

The group $Z^{4}(X)$ is a very interesting invariant recently studied in [13] and [40], which is known since 1962 to be nontrivial in general (see [3] and $[\mathbf{2 3}])$. In [13], this invariant is interpreted using unramified cohomology: If $A$ is an abelian group, we define sheaves $\mathcal{H}^{i}(A)$ on $X(\mathbb{C})_{\text {Zar }}$ by the formula

$$
\mathcal{H}^{i}(A)=R^{i} f_{*} A,
$$

where $f: X^{a n} \rightarrow X(\mathbb{C})_{Z a r}$ is the identity map on the set $X(\mathbb{C})$ changing the topology. Concretely, $\mathcal{H}^{i}(A)$ is the sheaf associated to the presheaf on $X(\mathbb{C})_{Z a r}$ which to $U \subset X$ associates $H_{B}^{i}(U, A)$. One then defines $H_{n r}^{i}(X, A)$ as $H^{0}\left(X_{Z a r}, \mathcal{H}^{i}(A)\right)$.

THEOREM 2.18. [13] If $X$ is rationally connected, then $Z^{4}(X)$ is isomorphic to the unramified cohomology group $H_{n r}^{3}(X, \mathbb{Q} / \mathbb{Z})$.

This makes $Z^{4}(X)$ the next degree analogue of the Artin-Mumford invariant, since it is easy to prove that, for rationally connected varieties, $\operatorname{Tors}\left(H_{B}^{3}(X, \mathbb{Z})\right)=H_{n r}^{2}(X, \mathbb{Q} / \mathbb{Z})$.

This interpretation of $Z^{4}(X)$ and the work of [11] allow to prove (cf. [13]).

TheOREM 2.19. The 6-dimensional quadric bundles $X$ over $\mathbb{P}^{3}$ built in [11] have $Z^{4}(X) \neq 0$.

On the other hand, it is proved in [40] that cubic fourfolds $X$ have $Z^{4}(X)$ trivial, which in turn can be interpreted using Theorem 2.19 by saying that they have trivial unramified degree 3 unramified cohomology.

2.3.3. Integral Hodge classes of degree $2 n-2$ and 1-cycles. The same arguments as above allow us to construct stable birational invariants from 1-cycles and their cycle classes.

Lemma 2.20. The group

$$
Z^{2 n-2}(X):=\operatorname{Hdg}^{2 n-2}(X, \mathbb{Z}) / H_{B}^{2 n-2}(X, \mathbb{Z})_{a l g}, n=\operatorname{dim} X
$$

is a stable birational invariant of smooth projective varieties.

REMARK 2.21. It is in fact more appropriate to write the degree $2 n-2$ cohomology as degree 2 homology in order to make clear that this group does not in fact depend on the dimension (the functoriality here is covariant). We will thus use the notation $Z_{2}(X)$ for $Z^{2 n-2}(X)$. 
In any dimension $n$, there are examples due to Kollár $[\mathbf{2 3}]$ of smooth projective high degree hypersurfaces $X$ in $\mathbb{P}^{n+1}$ with nontrivial $Z_{2}(X)$. On the other hand, there is some evidence that this group is trivial for rationally connected varieties.

THEOREM 2.22. (i) The group $Z_{2}(X)$ is trivial for rationally connected threefolds $[\mathbf{3 9}]$.

(ii) The group $Z_{2}(X)$ is trivial for Fano fourfolds $[\mathbf{2 0}]$.

(iii) Assuming the Tate conjecture for integral degree 2 Tate classes on surfaces defined over finite fields, the group $Z_{2}(X)$ is trivial for any smooth projective rationally connected variety over $\mathbb{C}[\mathbf{3 7}]$.

Another interesting stably birationally invariant group is the group $\operatorname{Griff}_{1}(X)$ of 1-cycles homologous to 0 modulo algebraic equivalence. A celebrated result of Griffiths $[\mathbf{1 7}]$ says that the group $\operatorname{Griff}_{1}(X)$ can be a nontorsion group, for example when $X$ is a very general quintic hypersurface in $\mathbb{P}_{\mathbb{C}}^{4}$.

Lemma 2.23. If $X$ is smooth projective rationally connected, the group $\operatorname{Griff}_{1}(X)$ is a torsion group.

Proof. There exists a dominant rational map $\phi: Y \times \mathbb{P}^{1} \rightarrow X$ mapping $Y \times 0$ to a point $x \in X$. We may assume that $\operatorname{dim} Y=n-1$. Then if $\tilde{\phi}: \widetilde{Y \times \mathbb{P}^{1}} \rightarrow X$ is a desingularization of $\phi, \tilde{\phi}$ has a finite nonzero degree $N$, and thus

$$
\tilde{\phi}_{*} \circ \tilde{\phi}^{*}=N I d
$$

on $\operatorname{Griff}_{1}(X)$. On the other hand, as $\operatorname{Griff}_{1}$ is stably birationally invariant, the map $i_{0 *}: \operatorname{Griff}_{1}(\tilde{Y}) \rightarrow \operatorname{Griff}_{1}\left(\widetilde{Y \times \mathbb{P}^{1}}\right)$ is surjective, where $\tilde{Y}$ is a desingularization of the proper transform of $Y \times 0$ in $\widetilde{Y \times \mathbb{P}^{1}}$. It follows that the map $\tilde{\phi}_{*}: \operatorname{Griff}_{1}\left(\widetilde{Y \times \mathbb{P}^{1}}\right) \rightarrow \operatorname{Griff}_{1}(X)$ vanishes identically, since $\tilde{\phi} \circ i_{0}$ is constant. Formula (6) then shows that $\operatorname{Griff}_{1}(X)$ is annihilated by $N$.

It is proved in [34] that the group $\operatorname{Griff}_{1}(X)$ is trivial if $X$ is a Fano complete intersection of index at least 2. I do not know if it is always trivial for a smooth projective rationally connected variety.

\section{Intermediate Jacobians}

\subsection{Intermediate Jacobians and Griffiths Abel-Jacobi map.}

3.1.1. Definition of the intermediate Jacobians. We consider the odd degree cohomology $H_{B}^{2 i-1}(X, \mathbb{Z}) /$ Torsion of a smooth complex variety of dimension $n$. The Hodge decomposition (3) provides the subspace

$$
F^{i} H_{B}^{2 i-1}(X, \mathbb{C}):=\oplus_{p+q=2 i-1, p \geq i} H^{p, q}(X) \subset H_{B}^{2 i-1}(X, \mathbb{C})
$$


and the Hodge symmetry implies that

$$
H_{B}^{2 i-1}(X, \mathbb{C})=F^{i} H_{B}^{2 i-1}(X, \mathbb{C}) \oplus \overline{F^{i} H_{B}^{2 i-1}(X, \mathbb{C})} .
$$

It follows that the natural map

$$
H_{B}^{2 i-1}(X, \mathbb{R}) \rightarrow H_{B}^{2 i-1}(X, \mathbb{C}) / F^{i} H_{B}^{2 i-1}(X, \mathbb{C})
$$

is an isomorphism. Thus the image of $H_{B}^{2 i-1}(X, \mathbb{Z}) /$ Torsion in $H_{B}^{2 i-1}(X, \mathbb{C}) /$ $F^{i} H_{B}^{2 i-1}(X, \mathbb{C})$ is a lattice and the quotient

$$
J^{2 i-1}(X)=H_{B}^{2 i-1}(X, \mathbb{C}) /\left(F^{i} H_{B}^{2 i-1}(X, \mathbb{C}) \oplus H_{B}^{2 i-1}(X, \mathbb{Z}) / \text { Torsion }\right)
$$

is a complex torus, called the $i$-th intermediate Jacobian of $X$.

It is not in general an abelian variety, except when $H^{p, q}(X)=0$ for $p+q=2 i-1$ and $p>i$ or $q>i$. In the later case, choosing an ample line bundle $\mathcal{L}$ on $X$ and denoting $l=c_{1}(\mathcal{L}) \in H_{B}^{2}(X, \mathbb{Z})$, the intersection pairing

$$
\langle\alpha, \beta\rangle_{l}:=\int_{X} l^{n-2 i+1} \smile \alpha \smile \beta
$$

on $H_{B}^{2 i-1}(X, \mathbb{Z}) /$ Torsion (conveniently modified in a way which takes into account the Lefschetz decomposition)) defines a polarization on $J^{2 i-1}(X)$ which makes it an abelian variety.

3.1.2. The Abel-Jacobi map. Let $X$ be a smooth complex projective variety of dimension $n$, and denote by $\mathcal{Z}^{i}(X)_{h o m}$ the group of cycles homologous to 0 . The Griffiths Abel-Jacobi map $\Phi_{X}: \mathcal{Z}^{i}(X)_{h o m} \rightarrow J^{2 i-1}(X)$ is defined as follows (this generalizes the Abel map): First of all, using Poincaré duality, we can see $J^{2 i-1}(X)$ as

$$
J^{2 i-1}(X)=F^{n-i+1} H_{B}^{2 n-2 i+1}(X, \mathbb{C})^{*} / H_{2 n-2 i+1, B}(X, \mathbb{Z}),
$$

where the map $H_{2 n-2 i+1, B}(X, \mathbb{Z}) \rightarrow F^{n-i+1} H_{B}^{2 n-2 i+1}(X, \mathbb{C})^{*}$ sends $a$ to $\int_{a}$. The image of this last map is called the group of periods.

Now let $Z$ be a cycle of codimension $i$ (hence real dimension $2 n-2 i$ ) which is homologous to 0 . Then there is a chain $\Gamma$ of real dimension $2 n-2 i+1$ such that $\partial \Gamma=Z$ (think for example to singular homology chains).

It is a key point that $\int_{\Gamma} \in F^{n-i+1} H_{B}^{2 n-2 i+1}(X, \mathbb{C})^{*}$ is well defined, even though $\Gamma$ is not closed. The key for that is to choose representatives of elements in $F^{n-i+1} H_{B}^{2 n-2 i+1}(X, \mathbb{C})$ which are closed forms of type $(2 n-$ $2 i+1,0)+\ldots+(n-i+1, n-i)$ and to apply the $d d$-bar lemma (see [43, 12.1.2]). Furthermore, $\Gamma$ is determined up to the addition of a closed chain, hence $\int_{\Gamma}$ is defined up to periods $\int_{a}, a \in H_{2 n-2 i+1, B}(X, \mathbb{Z})$. This gives us a point

$$
\Phi_{X}(Z)=\int_{\Gamma} \text { mod. periods } \in J^{2 i-1}(X) .
$$

The Abel-Jacobi map factors through rational equivalence (see [44, Lemma 9.19]), which provides

$$
\Phi_{X}: \mathrm{CH}^{i}(X)_{h o m} \rightarrow J^{2 i-1}(X) .
$$


3.1.3. Dimension 3: the Theta divisor . Let $X$ be a smooth projective threefold with $H^{1,0}(X)=H^{3,0}(X)=0$. Then $J^{3}(X)$ is canonically a principally polarized abelian variety. We use for this the unimodular intersection pairing $\langle,\rangle_{X}$ on $H_{B}^{3}(X, \mathbb{Z}) /$ Torsion. By our hypothesis, this skew-symmetric pairing satisfies the Riemann relations

$$
\begin{gathered}
\langle\alpha, \beta\rangle_{X}=0, \forall \alpha, \beta \in F^{2} H^{3}(X)=H^{2,1}(X), \\
-\iota\langle\alpha, \bar{\alpha}\rangle_{X}>0, \forall 0 \neq \alpha \in H^{2,1}(X) .
\end{gathered}
$$

These conditions guarantee that minus the intersection form $\langle,\rangle_{X}$, seen as an element of the group $\bigwedge^{2} H_{1}\left(J^{3}(X), \mathbb{Z}\right)^{*}=H^{2}\left(J^{3}(X), \mathbb{Z}\right)$, is the first Chern class of an ample line bundle on $J^{3}(X)$, which is thus an abelian variety. The fact that the pairing is unimodular guarantees that this line bundle (which is well-defined up to translation) has a unique nonzero section up to scalar multiples. This shows that $J^{3}(X)$ has a Theta divisor, determined up to translation, and defined as the zero set of this unique section.

\subsection{Codimension 1 cycles.}

3.2.1. Abel's theorem. In the case of codimension 1 cycles, the following result is one version of Abel's theorem.

Theorem 3.1. The Abel-Jacobi map $\Phi_{X}: \mathrm{CH}^{1}(X)_{h o m} \rightarrow J^{1}(X)$ is an isomorphism.

This is proved by looking at the beginning of the long exact sequence associated to the exponential exacte sequence (4): This provides us (using $\mathrm{CH}^{1}(X)=\operatorname{Pic} X=\operatorname{Pic} X^{a n}$ and $\left.\mathrm{CH}^{1}(X)_{h o m}=\operatorname{Ker} c_{1} \subset \operatorname{Pic} X^{a n}\right)$

$$
0 \rightarrow H^{1}(X, \mathbb{Z}) \rightarrow H^{1}\left(X, \mathcal{O}_{X}\right) \rightarrow \mathrm{CH}^{1}(X)_{\text {hom }} \rightarrow 0 .
$$

Hence we get, using $H^{0,1}(X)=H^{1}\left(X, \mathcal{O}_{X}\right)$, an isomorphism $J^{1}(X)=$ $\mathrm{CH}^{1}(X)_{h o m}$ and the proof is concluded by showing that this is the inverse of the Abel-Jacobi map (see [43, Proposition 12.7]).

3.2.2. Existence of a universal divisor. By the previous sections, we know that if $X$ is smooth and projective over $\mathbb{C}, J^{1}(X)$ is always an abelian variety and furthermore it parameterizes divisors homologous to 0 on $X$ modulo linear equivalence, or equivalently topologically trivial line bundles on $X$. In fact much more is true.

TheOREM 3.2. There exists a line bundle $\mathcal{P}$ on $J^{1}(X) \times X$ which has the following property: For any $t \in J^{1}(X)$ the divisor $\mathcal{D}_{t} \in \mathrm{CH}^{1}(X)$ is homologous to 0 and $\Phi_{X}\left(\mathcal{D}_{t}\right)=t$.

Such a divisor is called the universal divisor of $X$ or the Poincare divisor if $X$ is an abelian variety. It is not unique but can be normalized by fixing a point $x \in X$ and imposing the condition that $\mathcal{P}_{\mid J^{1}(X) \times\{x\}}$ is trivial. 
Sketch OF PRoOF OF TheOREM 3.2. There are several approaches to the existence of the universal divisor. One is transcendental and is based on the observation that there is a natural degree 2 integral Hodge class on $J^{1}(X) \times X$, namely the one which induces the canonical isomorphism

$$
H_{1}\left(J^{1}(X), \mathbb{Z}\right) \cong H_{B}^{1}(X, \mathbb{Z}) .
$$

It suffices then to apply the Lefschetz $(1,1)$-theorem.

The second approach is purely algebraic: One observes that if one chooses a complete family

$$
\mathcal{D} \subset \mathcal{M} \times X
$$

of sufficiently ample hypersurfaces on $X$, the Abel-Jacobi map

$$
\begin{gathered}
\Phi_{\mathcal{D}}: \mathcal{M} \rightarrow J^{1}(X) \\
m \mapsto \Phi_{X}^{1}\left(\mathcal{D}_{m}-\mathcal{D}_{0}\right),
\end{gathered}
$$

where 0 is a chosen base point on $\mathcal{M}$, makes $\mathcal{M}$ into a projective bundle over $J^{1}(X)$. This is a honest projective bundle, and not a Brauer-Severi variety, because by Abel's theorem, the fibers of $\Phi_{\mathcal{D}}$ are linear systems $\left|\mathcal{D}_{t}\right|$ and thus fixing a point $x \in X$, we have a codimension 1 projective subbundle consisting of divisors passing through $x$. Hence there is a rational section $\sigma$ of the morphism $\Phi_{\mathcal{D}}$. Then $\left(\sigma, I d_{X}\right)^{*} \mathcal{D}$ provides the desired universal divisor.

3.2.3. Codimension 2 cycles. Let $X$ be a rationally connected smooth projective variety. Then $H^{3,0}(X)=0$ by Lemma 2.2 and thus $J^{3}(X)$ is an abelian variety. The following result is due to Bloch and Srinivas $[\mathbf{8}]$ (see also $[\mathbf{2 9}])$ :

Theorem 3.3. We have $\mathrm{CH}^{2}(X)_{\text {hom }}=\mathrm{CH}^{2}(X)_{\text {alg }}$ and the Abel-Jacobi map

is an isomorphism.

$$
\Phi_{X}: \mathrm{CH}^{2}(X)_{h o m} \rightarrow J^{3}(X)
$$

Note that the left hand side is only a group, while the right hand side is an algebraic group, but it makes sense to say that $\Phi_{X}$ is algebraic. Indeed the meaning is the following: For any smooth algebraic variety $M$, and any codimension 2 cycle $\mathcal{Z} \in \mathrm{CH}^{2}(M \times X)$ such that the restricted cycles $\mathcal{Z}_{m}, m \in M$, are homologous to 0 on $X$, the map

$$
\Phi_{\mathcal{Z}}: M \rightarrow J^{3}(X), m \mapsto \Phi_{X}\left(\mathcal{Z}_{m}\right),
$$

is a morphism of algebraic varieties. Theorem 3.3 is a beautiful analogue of Abel's theorem for divisors and shows that codimension 2 cycles on rationally connected varieties behave very much as codimension 1 cycles on any variety. Of course the assumption that $X$ is rationally connected is crucial here (see $[\mathbf{2 8}],[\mathbf{1 7}]$ for various pathologies which appear otherwise).

However, a question remains open, namely: 
QUESTION 3.4. Let $X$ be smooth projective rationally connected over $\mathbb{C}$. Does there exist a universal codimension 2 cycle on $J^{3}(X) \times X$, that is $\mathcal{Z} \in \mathrm{CH}^{2}\left(J^{3} \times X\right)$ such that the restricted cycles $\mathcal{Z}_{t} \in \mathrm{CH}^{2}(X), t \in J^{3}(X)$, are cohomologous to 0 and the morphism

$$
\Phi_{\mathcal{Z}}: J^{3}(X) \rightarrow J^{3}(X)
$$

is the identity?

This question, which we will answer negatively in next section, was first asked and studied in [38]. One motivation is the following fact:

Proposition 3.5. The existence of a universal codimension 2 cycle is a stably birationally invariant property.

Proof. Indeed, consider first invariance under the substitution $X \rightsquigarrow$ $X \times \mathbb{P}^{r}, r \geq 1$. Then we have

$$
J^{3}\left(X \times \mathbb{P}^{r}\right)=J^{3}(X) \oplus J^{1}(X),
$$

where the inclusion $J^{1}(X) \rightarrow J^{3}(X)$ is given by $\alpha \mapsto p r_{2}^{*} h \cdot p r_{1}^{*} \alpha$. (We use the fact that Hodge classes act on Jacobians via their cup-product on odd degree cohomology.) Now we also have

$$
\mathrm{CH}^{2}\left(X \times \mathbb{P}^{r}\right)_{\text {hom }}=\mathrm{CH}^{2}(X)_{\text {hom }} \oplus \mathrm{CH}^{1}(X)_{\text {hom }} .
$$

Using the functoriality of the Abel-Jacobi map with respect to pull-back and intersection with a cycle class (see $[\mathbf{4 4}, 9.2 .4]$ ), and the fact that there exists a universal codimension 1 divisor on $J^{1}(X) \times X$, we easily conclude that $X$ has a universal codimension 2 cycle if and only if $X \times \mathbb{P}^{r}$ does. We next have to check invariance under birational maps, which is very similar as before. As usual, using the Hironaka desingularization theorem, we reduce to proving that the property is invariant under blow-ups. Next if $Z \subset X$ is the inclusion of a smooth projective subvariety, we have

$$
J^{3}\left(\widetilde{X}_{Z}\right)=\tau^{*} J^{3}(X) \oplus J^{1}(Z),
$$

where the map $J^{1}(Z) \rightarrow J^{3}\left(\widetilde{X}_{Z}\right)$ is given by $j_{E *} \circ \tau_{E}^{*}$. Similarly, we have

$$
\mathrm{CH}^{2}\left(\widetilde{X}_{Z}\right)_{h o m}=\mathrm{CH}^{2}(X)_{h o m} \oplus \mathrm{CH}^{1}(Z)_{h o m},
$$

where the maps are given by the same formulas on the level of algebraic cycles. The Abel-Jacobi map is compatible with these decompositions. Then as before we use the fact that $Z$ has a universal divisor and the compatibility of the Abel-Jacobi map with the various operations to conclude that $\widetilde{X}_{Z}$ has a universal codimension 2 cycle if and only if $X$ does. More precisely, if $X$ has a universal codimension 2 cycle $\mathcal{Z} \in \mathrm{CH}^{2}\left(J^{3}(X) \times X\right)$ and $\mathcal{D}$ is a universal divisor for $Z$, then

$$
\mathcal{Z}^{\prime}:=(\alpha, \tau)^{*} \mathcal{Z}+\left(I_{J^{3}\left(\widetilde{X}_{Z}\right)}, j_{E}\right)_{*}\left(\beta, \tau_{E}\right)^{*} \mathcal{D}
$$

is a universal codimension 2 cycle for $\widetilde{X}_{Z}$, where

$$
\alpha: J^{3}\left(\tilde{X}_{Z}\right) \rightarrow J^{3}(X), \beta: J^{3}\left(\tilde{X}_{Z}\right) \rightarrow J^{1}(Z)
$$


are the two projections induced by (8).

It will be proved in Section 4.3.3 that Question 3.4 above does not have in general an affirmative answer for some unirational threefolds with trivial Artin-Mumford invariant. Hence by Proposition 3.5, the existence of a universal codimension 2 cycle is an effective necessary criterion for the stable rationality of unirational varieties.

3.3. Cubic threefolds. We first review in this section the beautiful work [10] which provides a strong necessary condition for rationality of a smooth projective threefold. We will finally discuss a generalization discovered in $[\mathbf{3 8}],[\mathbf{4 2}]$, concerning stable rationality of smooth projective threefolds.

3.3.1. Clemens-Griffiths criterion. Let $X$ be a smooth complex projective 3 -fold with $H^{3,0}(X)=H^{1,0}(X)=0$. We mentioned that $J^{3}(X)$ is then canonically a principally polarized abelian variety. The Clemens-Griffiths criterion for rationality is the following:

Theorem 3.6. If $X$ is rational, $\left(J^{3}(X), \Theta_{X}\right)$ is a direct product of Jacobians of smooth curves $\left(J\left(C_{i}\right), \Theta_{C_{i}}\right)$.

PROOF. If $X$ is rational, there is a diagram

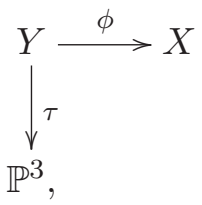

where $\phi$ is a projective morphism of degree 1 and $\tau$ is a composition of blowups along smooth connected centers. It follows then from the computation of the cohomology and Hodge structures of a blow-up that $\left(J^{3}(Y), \Theta_{Y}\right)$ is, as a ppav, a product

$$
\left(J^{3}(Y), \Theta_{Y}\right)=\prod\left(J\left(C_{i}\right), \Theta_{C_{i}}\right),
$$

where the $C_{i}$ 's are the curves blown-up by $\tau$ (blowing-up a point does not affect $J^{3}$ ). It remains to prove that the same thing happens for $X$. The point is that we have the pull-back map $\phi^{*}: H^{3}(X, \mathbb{Z}) \rightarrow H^{3}(Y, \mathbb{Z})$ which is an injective morphism of Hodge structures (with left inverse $\phi_{*}$ ), and is compatible with intersection pairing since $\operatorname{deg} \phi=1$. It follows that

$$
\left(J^{3}(Y), \Theta_{Y}\right)=\left(J^{3}(X), \Theta_{X}\right) \times\left(B, \Theta_{B}\right),
$$

where $\left(B, \Theta_{B}\right)$ is a principally polarized abelian variety. We now use two facts:

1) The decomposition of a principally polarized abelian variety as a product of irreducible principally polarized abelian varieties is unique up to permutation of the factors.

2) The Jacobian of a smooth connected curve is irreducible as a principally polarized abelian variety. 
The second point is an immediate consequence of Riemann's description (see $\left[\mathbf{1}\right.$, p. 27]) of the Theta divisor of $J^{1}(C)$, where $C$ is a smooth projective connected curve of genus $g$, as the image in $J^{1}(C)$ of $C^{(g-1)}$ via the Abel map (a base-point being chosen on $C$ ). From 1) and (11), we deduce that $\left(J^{3}(X), \Theta_{X}\right)$ is the product of some of the irreducible ppav's appearing in the decomposition of $\left(J^{3}(Y), \Theta_{Y}\right)$. From (10) and 2), we deduce that the irreducible ppav's appearing in the decomposition of $\left(J^{3}(Y), \Theta_{Y}\right)$ are Jacobians of smooth curves.

3.3.2. Application to the cubic threefold. The Clemens-Griffiths criterion has been successfully applied to prove the irrationality of many rationally connected threefolds, particularly conic bundles (see [6], [9]). The model for these results was first provided by Clemens and Griffiths [10], who proved

TheOREM 3.7. Let $X$ be a smooth cubic hypersurface in $\mathbb{P}^{4}$. Then $X$ is not rational.

Note that $X$ is unirational, as all smooth cubic hypersurfaces of dimension $\geq 2$ over an algebraically closed field are. Indeed, choose a line $\Delta \subset X$. Consider the projective bundle $\mathbb{P}\left(T_{X \mid \Delta}\right)$. This is clearly a rational variety and it dominates rationally $X$ by the map which to $(x, u), x \in \Delta, u \in \mathbb{P}\left(T_{X, x}\right)$ associates the residual point of the intersection with $X$ of the line passing through $x$ with tangent direction $u$.

Theorem 3.7 is deduced from Theorem 3.6 and a beautiful analysis of $J^{3}(X)$. It is proved that this is an irreducible ppav of dimension 5 and that the singular locus of its Theta divisor consists of a single point. As the singular locus of the Theta divisor of a smooth projective curve has codimension $\leq 4$ by Riemann singularity theorem [1, p. 226], this is enough to conclude.

3.3.3. A generalized Clemens-Griffiths criterion. Let $(A, \Theta)$ be a principally polarized abelian variety of dimension $g$. The class $\theta=[\Theta] \in H_{B}^{2}(A, \mathbb{Z})$ has the property that the class $\theta^{g-1} /(g-1)$ ! is a degree $2 g-2$ integral Hodge class on $A$. Of course the class $\theta^{g-1}$ is algebraic, but it is not clear if $\theta^{g-1} /(g-1)$ !, which is called the minimal class, is also algebraic, although no counterexample is known.

By Matsusaka work's [27], one has the following characterization of products of Jacobians.

TheOREm 3.8. A ppav $(A, \Theta)$ is a product of Jacobians if and only if the minimal class is the class of an effective 1-cycle in $A$.

Using this theorem, one can rephrase Clemens-Griffiths' theorem 3.6 by saying that if $X$ is rational, there is an effective 1-cycle in the minimal class of $\left(J^{3}(X), \Theta_{X}\right)$.

The following result, which will be proved in a much more general form in Section 4.2.3 has been obtained in [42]. 
TheOREM 3.9. Let $X$ be a stably rational threefold. Then the minimal class of $\left(J^{3}(X), \Theta_{X}\right)$ is algebraic.

It is a completely open problem whether this criterion is restrictive or not. In particular, it is not known if it is satisfied by the general cubic threefold (and it is not known if a general (and in fact, any) cubic threefold is stably rational or not).

\section{Decomposition of the diagonal}

4.1. Generalities. We are now going to exploit an invariant which has been introduced and studied in a cohomological form in $[\mathbf{3 8}]$ and in a Chow-theoretic form in [4]. This invariant is clearly interesting for the stable Lüroth problem, because as we will see below it controls the "classical" invariants that we described in the previous sections, namely the ArtinMumford invariant, and the invariant $Z^{4}(X)$. The main novelty that we have discovered in [36] is the fact that it is actually more powerful and can be used to show stable irrationality of many classes of unirational varieties, which are much less exotic than the Artin-Mumford or Colliot-Thélène-Ojanguren examples.

4.1.1. Definitions. Let $X$ be a smooth projective variety of dimension $n$ over a field $K$ and let $x \in X(K)$.

Definition 4.1. (i) We say that $X$ has a Chow decomposition of the diagonal if

$$
\Delta_{X}=X \times x+Z \text { in } \mathrm{CH}^{n}(X \times X),
$$

where $Z$ is a cycle supported on $D \times X$ for some closed proper algebraic subset $D \varsubsetneqq X$.

(ii) If $K=\mathbb{C}, X$ is said to have a cohomological decomposition of the diagonal if

$$
\left[\Delta_{X}\right]=[X \times x]+[Z] \text { in } H_{B}^{2 n}(X \times X, \mathbb{Z}),
$$

where $Z$ is as above.

Lemma 4.2. If $X$ has a Chow decomposition of the diagonal, then $\mathrm{CH}_{0}(X)=\mathbb{Z}$. In particular, all $K$-points of $X$ are rationally equivalent.

Proof. Indeed, both sides of (12) act on $\mathrm{CH}_{0}(X)$ and as the diagonal cycle acts as the identity, we get for any $z \in \mathrm{CH}_{0}(X)$ :

$$
z=(X \times x)_{*} z+Z_{*} z \text { in } \mathrm{CH}_{0}(X) .
$$

But as $Z$ is supported over $D \times X$, where $D \varsubsetneqq X$, we get $Z_{*} z=0$. On the other hand, it is obvious that $(X \times x)_{*} z=(\operatorname{deg} z) x$ in $\mathrm{CH}_{0}(X)$. Hence $z=(\operatorname{deg} z) x$ in $\mathrm{CH}_{0}(X)$.

When the field $K$ is big, like $\mathbb{C}$, this lemma has an almost converse which is due to Bloch and Srinivas and is at the origin of the notion of decomposition of the diagonal. 
TheOREm 4.3. Let $X$ be a variety over $\mathbb{C}$. Then if $\mathrm{CH}_{0}(X)=\mathbb{Z}, X$ admits a Chow decomposition of the diagonal with $\mathbb{Q}$-coefficients, that is, for some integer $N>0$

$$
N \Delta_{X}=N(X \times x)+Z \text { in } \mathrm{CH}^{n}(X \times X),
$$

where $Z$ is a cycle supported on $D \times X$ for some closed proper algebraic subset $D \varsubsetneqq X$.

Corollary 4.4. A rationally connected variety over $\mathbb{C}$ has a Chow decomposition of the diagonal with $\mathbb{Q}$-coefficients.

This last fact can in fact be proved directly and is true over any field of characteristic 0 .

A DIRECT PROOF OF COROLlary 4.4. Indeed there exists a rational dominating map

$$
Y \times \mathbb{P}^{1} \rightarrow X
$$

contracting $Y \times 0$ to a point $x \in X$. We may assume $\operatorname{dim} Y=n-1$. Let $\phi: \widetilde{Y \times \mathbb{P}^{1}} \rightarrow X$ be a desingularization of this rational map and let $N:=\operatorname{deg} \phi$. It is then clear that $(\phi, \phi)_{*} \Delta_{\widetilde{Y \times \mathbb{P} 1}}=N \Delta_{X}$ in $\mathrm{CH}^{n}(X \times X)$. Now the diagonal of $\widetilde{Y \times \mathbb{P}^{1}}$ decomposes as the sum of a term $Z_{0}$ supported on $E \times E$, where $E$ is the exceptional divisor of the blow-up map $\widetilde{Y \times \mathbb{P}^{1}} \rightarrow$ $Y \times \mathbb{P}^{1}$, and of the pull-back of the diagonal of $Y \times \mathbb{P}^{1}$. For the latter, we use the fact that $\Delta_{\mathbb{P}^{1}}=\mathbb{P}^{1} \times 0+0 \times \mathbb{P}^{1}$, and we conclude that $Y \times \mathbb{P}^{1}$ is the sum of a term $Z_{1}$ supported on $Y \times \mathbb{P}^{1} \times Y \times 0$ and of a term $Z_{2}$ supported on $Y \times 0 \times Y \times \mathbb{P}^{1}$. As $Y \times 0$ is contracted to $x$ by $\phi,(\phi, \phi)_{*} Z_{1}$ is supported on $X \times 0$ and $(\phi, \phi)_{*} Z_{2}$ is supported on $0 \times X$. Finally $(\phi, \phi)_{*}\left(Z_{0}\right)$ is supported over $\phi(E) \times \phi(E)$. Putting everything together we constructed a Chow decomposition of $N \Delta_{X}$.

4.1.2. Relation to the universal $\mathrm{CH}_{0}$ group. Following [4], we now formulate the notion of having a Chow decomposition of the diagonal in the following form, involving the study of $X$ over surfields $L$ of $K$. This viewpoint makes very clear why a variety defined over an algebraically closed field may not have a decomposition of the diagonal, even if it is rationally connected.

Definition 4.5. The variety $X$ has universally trivial $\mathrm{CH}_{0}$ group if for any field $L$ containing $K$, one has $\mathrm{CH}_{0}\left(X_{L}\right)=\mathbb{Z} x_{L}$, where $x$ is a $K$-point of $X$.

Let us explain the proof of the following equivalence proved in [4].

Lemma 4.6. Having a Chow decomposition of the diagonal and having universally trivial $\mathrm{CH}_{0}$ group are equivalent properties. 
Proof. First of all, if $X$ admits a decomposition of the diagonal over $K$ for some point $x \in X(K)$, so does $X_{L}$, hence $\mathrm{CH}_{0}\left(X_{L}\right)=\mathbb{Z} x_{L}$ by Lemma 4.2. In the other direction, assume $\mathrm{CH}_{0}(X)$ is universally trivial and take for $L$ the function field $K(X)$ of $X$. Then we have the generic point $\delta_{L} \in \mathrm{CH}_{0}\left(X_{L}\right)=\mathrm{CH}^{n}\left(X_{L}\right)$. Clearly its class in $\mathrm{CH}^{n}\left(X_{L}\right)$ is the restriction to $\operatorname{Spec}(K(X)) \times X$ of the diagonal $\Delta_{X} \in \mathrm{CH}^{n}(X \times X)$. The fact that $\delta_{L}$ has the same class as $x_{L}$ in $\mathrm{CH}_{0}\left(X_{L}\right)$ thus says that the difference $\Delta_{X}-X \times x$ vanishes in $\mathrm{CH}^{n}\left(X_{L}\right)$, hence that for some dense Zariski open set $U$ of $X$, $\Delta_{X}-X \times x$ vanishes in $\mathrm{CH}^{n}(U \times X)$. By the localization exact sequence (1), we conclude that $\Delta_{X}-X \times x$ is rationally equivalent to a cycle supported on $D \times X$, where $D:=X \backslash U$.

4.1.3. Stable birational invariance. The following fact was our motivation in $[\mathbf{3 8}]$ to study the decomposition of the diagonal.

Proposition 4.7. The existence of a (Chow-theoretic or cohomological) decomposition of the diagonal is a stably birationally invariant property of smooth projective varieties.

Proof. First of all, if $X \times Y$ admits a decomposition of the diagonal, where $Y$ is smooth projective, so does $X$. Indeed, we have $\Delta_{X}=$ $\operatorname{pr}_{X \times X *}\left(\Delta_{X \times Y \mid X \times X \times Y \times y}\right)$, where $p r_{X \times X}$ is the projection from $X \times X \times Y$ to $X \times X$. This now implies that $X$ admits a decomposition of the diagonal if and only $X \times \mathbb{P}^{r}$ does. For this, we just have to show that if $X$ admits a decomposition of the diagonal, so does $X \times \mathbb{P}^{r}$. But this immediately follows from the fact that $\mathbb{P}_{K}^{r}$ admits a Chow decomposition of the diagonal. Namely, if $h=c_{1}\left(\mathcal{O}_{\mathbb{P}_{K}^{r}}(1)\right) \in \mathrm{CH}^{1}\left(\mathbb{P}_{K}^{r}\right)$, we have $\Delta_{\mathbb{P}_{K}^{r}}=\sum_{i=0}^{r} p_{1}^{*} h^{i} \cdot p_{2}^{*} h^{r-i}$ and $h^{r}$ is the class of any $K$-point of $\mathbb{P}_{K}^{r}$, while $p_{1}^{*} h^{i} \cdot p_{2}^{*} h^{r-i}$ is clearly supported in $D \times X$ for some closed algebraic subset $D \varsubsetneqq X$ if $i>0$.

For the birational invariance, let us use the desingularization theorem in the weak form saying that if $\phi: X \rightarrow Y$ is a birational map, then there exist a smooth projective variety $X^{\prime}$, and two morphisms $\tau: X^{\prime} \rightarrow X$, $\tau^{\prime}: X^{\prime} \rightarrow Y$ of degree 1 such that $\phi=\tau^{\prime} \circ \tau^{-1}$. This reduces the birational invariance of the property to the case of birational morphisms $\tau: X^{\prime} \rightarrow X$. But then we have

$$
\begin{gathered}
(\tau, \tau)_{*}\left(\Delta_{X^{\prime}}\right)=\Delta_{X} \text { in } \mathrm{CH}^{n}(X \times X), \\
(\tau, \tau)_{*}\left(\left[\Delta_{X^{\prime}}\right]\right)=\left[\Delta_{X}\right] \text { in } H_{B}^{2 n}(X \times X, \mathbb{Z}),
\end{gathered}
$$

and for some cycle $Z^{\prime}$ supported on $E \times E$, where $E$ is the exceptional divisor of $\tau$,

$$
\begin{gathered}
(\tau, \tau)^{*}\left(\Delta_{X}\right)=\Delta_{X^{\prime}}+Z^{\prime} \text { in } \mathrm{CH}^{n}\left(X^{\prime} \times X^{\prime}\right), \\
(\tau, \tau)^{*}\left(\left[\Delta_{X}\right]\right)=\left[\Delta_{X^{\prime}}\right]+\left[Z^{\prime}\right] \text { in } H_{B}^{2 n}\left(X^{\prime} \times X^{\prime}, \mathbb{Z}\right) .
\end{gathered}
$$

These formulas immediately imply that $X$ has a decomposition of the diagonal if and only if $X^{\prime}$ does since the cycle $Z^{\prime}$ does not dominate $X^{\prime}$ by the first projection. 
REMARK 4.8. For the Chow-theoretic version, one could use as well the stable birational invariance of $\mathrm{CH}_{0}$ and Lemma 4.6.

4.2. Consequences. We are going to describe in this section three consequences of the existence of a cohomological decomposition of the diagonal. More consequences can be obtained using a Chow-theoretic decomposition of the diagonal.

4.2.1. Generalized Artin-Mumford invariants. Let us start with the Artin-Mumford invariant and the invariant $Z^{4}(X)$. As we noticed, these groups respectively identify, for a rationally connected variety over $\mathbb{C}$, with the groups $H_{n r}^{2}(X, \mathbb{Q} / \mathbb{Z})$ and $H_{n r}^{3}(X, \mathbb{Q} / \mathbb{Z})$.

Proposition 4.9. Let $X$ be smooth projective of dimension $n$. Then if $X$ admits a cohomological decomposition of the diagonal, the groups $Z^{4}(X)=\operatorname{Hdg}^{4}(X, \mathbb{Z}) / H_{B}^{4}(X, \mathbb{Z})_{\text {alg }}$ and Tors $H_{B}^{3}(X, \mathbb{Z})$ vanish.

Proof. By assumption, there exist a closed proper algebraic subset $D \subset X$ and a cycle $Z$ supported on $D \times X$ such that

$$
\left[\Delta_{X}\right]=[X \times x]+[Z] \text { in } H^{2 n}(X \times X, \mathbb{Z}) .
$$

We now observe that we can choose $D$ to be a divisor having for each component $Z_{i}$ of the support of $Z$ a component $D_{i}$ such that $D_{i} \times X$ contains $Z_{i}$ and is generically smooth along it. Then if $j: \widetilde{D} \rightarrow D \hookrightarrow X$ is a desingularization of $D$, the cycle $Z$ lifts to a cycle $\widetilde{Z} \in \mathrm{CH}^{n-1}(\widetilde{D} \times X)$. Equation (14) then rewrites as

$$
\left[\Delta_{X}\right]=[X \times x]+\left(j, I d_{X}\right)_{*}[\widetilde{Z}] \text { in } H_{B}^{2 n}(X \times X, \mathbb{Z}) .
$$

We now let both sides of $(15)$ act on $H_{B}^{*}(X, \mathbb{Z})$. If $\alpha \in H^{k}(X, \mathbb{Z})$ with $k>0$, then $[X \times x]^{*} \alpha=0$, and we thus get

$$
\alpha=j_{*}\left([\widetilde{Z}]^{*} \alpha\right) \text { in } H_{B}^{k}(X, \mathbb{Z}) .
$$

If now $\alpha \in \operatorname{Tors} H_{B}^{3}(X, \mathbb{Z})$,

$$
[\widetilde{Z}]^{*} \alpha \in \operatorname{Tors} H_{B}^{1}(\widetilde{D}, \mathbb{Z})=0
$$

and we thus conclude from (16) that $\alpha=0$. Similarly, if $\alpha \in \operatorname{Hdg}^{4}(X, \mathbb{Z})$ then

$$
[\widetilde{Z}]^{*} \alpha \in \operatorname{Hdg}^{2}(\widetilde{D}, \mathbb{Z})=H_{B}^{2}(\widetilde{D}, \mathbb{Z})_{a l g}
$$

and we thus conclude from $(16)$ that $\alpha \in H_{B}^{4}(X, \mathbb{Z})_{\text {alg }}$.

4.2.2. Universal codimension 2 cycle. We observed in Section 3.2.3 that if $X$ is a stably rational variety, there exists a universal codimension 2 cycle on $J^{3}(X) \times X$. The following gives a strengthened version of this result.

Proposition 4.10. Let $X$ be smooth projective complex. Then if $X$ admits a cohomological decomposition of the diagonal, one has $H^{0, i}(X)=0$ for $i>0$, the Abel-Jacobi map

$$
\Phi_{X}: \mathrm{CH}^{2}(X)_{h o m} \rightarrow J^{3}(X)
$$


is surjective, and there exists a universal codimension 2 cycle on $J^{3}(X) \times X$.

REMARK 4.11. The existence of a universal codimension 2 cycle with $\mathbb{Q}$-coefficients on $J^{3}(X) \times X$ follows from the fact that the Abel-Jacobi map is surjective. Indeed, this implies that for some smooth projective variety $B$ and some codimension 2 cycle $\mathcal{Z} \in \mathrm{CH}^{2}(B \times X)$ such that $\mathcal{Z}_{b}$ is homologous to 0 on $X$ for any $b \in B$, the morphism

$$
\Phi_{\mathcal{Z}}: B \rightarrow J^{3}(X)
$$

is surjective. But then we may assume this morphism is generically finite of degree $N$, and the cycle

$$
\mathcal{Z}^{\prime}:=\left(\Phi_{\mathcal{Z}}, I d_{X}\right)_{*} \mathcal{Z} \in \mathrm{CH}^{2}\left(J^{3}(X) \times X\right)
$$

satisfies

$$
\Phi_{\mathcal{Z}^{\prime}}=N I d_{J^{3}(X)}
$$

Proof of Proposition 4.10. The fact that the existence of a cohomological decomposition diagonal implies the vanishing of the groups $H^{i}\left(X, \mathcal{O}_{X}\right)$ for $i>0$ is proved in [8] and uses only a cohomological decomposition of the diagonal with $\mathbb{Q}$-coefficients. The surjectivity of the Abel-Jacobi map $\Phi_{X}: \mathrm{CH}^{2}(X) \rightarrow J^{3}(X)$ is also proved in loc. cit. assuming only a cohomological decomposition of the diagonal with $\mathbb{Q}$-coefficients. It will be in fact a consequence of the existence of a universal codimension 2 cycle. We prove now the last statement. As in the previous proof, the existence of a cohomological decomposition of the diagonal provides us with a morphism $j: \widetilde{D} \rightarrow X$ from a smooth variety $\widetilde{D}$ of dimension $n-1$, and of a cycle $\widetilde{Z} \in \mathrm{CH}^{n-1}(\widetilde{D})$ such that

$$
\left[\Delta_{X}\right]=[X \times x]+\left(j, I d_{X}\right)_{*}[\widetilde{Z}] \text { in } H_{B}^{2 n}(X \times X, \mathbb{Z}) .
$$

This equality gives us for any $\alpha \in H_{B}^{3}(X, \mathbb{Z})$

$$
\alpha=j_{*}\left([\widetilde{Z}]^{*} \alpha\right) \text { in } H_{B}^{3}(X, \mathbb{Z}) .
$$

The morphisms $j_{*}: H_{B}^{1}(\widetilde{D}, \mathbb{Z}) \rightarrow H_{B}^{3}(X, \mathbb{Z})$ and $[\widetilde{Z}]^{*}: H_{B}^{3}(X, \mathbb{Z}) \rightarrow$ $H_{B}^{1}(\widetilde{D}, \mathbb{Z})$ are morphisms of Hodge structures of odd weight, hence induce morphisms of the corresponding intermediate Jacobians:

$$
j_{*}: J^{1}(\widetilde{D}) \rightarrow J^{3}(X),[\widetilde{Z}]^{*}: J^{3}(X) \rightarrow J^{1}(\widetilde{D}),
$$

and we have

$$
j_{*} \circ[\widetilde{Z}]^{*}=I d_{J^{3}(X)} .
$$

By Theorem 3.2, there exists a universal divisor $\mathcal{D}$ on $J^{1}(\widetilde{D}) \times \widetilde{D}$. Denoting by $\gamma$ the morphism $[\widetilde{Z}]^{*}$, we can consider the codimension 2 cycle on $J^{3}(X) \times X$ defined by

$$
\mathcal{Z}:=\left(I_{J^{3}(X)}, j\right)_{*}\left(\left(\gamma, I d_{\widetilde{D}}\right)^{*} \mathcal{D}\right) \in \mathrm{CH}^{2}\left(J^{3}(X) \times X\right) .
$$

It is immediate to check using (19) that $\mathcal{Z}$ is a universal codimension 2 cycle. 
4.2.3. Minimal class on intermediate Jacobian. We now concentrate on the case where $X$ has dimension 3 and satisfies $h^{0, i}(X)=0$ for $i>0$. Recall from Section 3.1.3 that in this case $J^{3}(X)$ is a principally polarized abelian variety with polarizing class $\theta$ and minimal class $\theta^{g-1} /(g-1) ! \in$ $H^{2 g-2}\left(J^{3}(X), \mathbb{Z}\right), g=\operatorname{dim} J^{3}(X)$. The following result is proved in [42].

THEOREM 4.12. A smooth projective threefold $X$ with $h^{0, i}(X)=0$ for $i>0$ admits a cohomological decomposition of the diagonal if and only if the following four conditions are satisfied:

(i) $\operatorname{Tors} H_{B}^{*}(X, \mathbb{Z})=0$.

(ii) $Z^{4}(X)=0$.

(iii) There exists a universal codimension 2 cycle on $J^{3}(X) \times X$.

(iv) The minimal class $\theta^{g-1} /(g-1)$ ! is algebraic on $J^{3}(X)$.

In the case where $X$ is rationally connected, condition (ii) is automatically satisfied by [39]. The fact that (i), (ii) and (iii) are necessary is essentially proved in Propositions 4.9 and 4.10. The necessity of condition (iv) is more difficult and proved in [42, Theorem 4.1]. In the other direction, the existence of a cohomological decomposition of the diagonal assuming (i) to (iv) is proved in [38, Theorem 4.9].

\subsection{Non-existence.}

4.3.1. The degeneration theorem. The following result is proved in [36].

ThEOREM 4.13. Let $\pi: \mathcal{X} \rightarrow B$ be a flat projective morphism of relative dimension $n \geq 2$, where $B$ is a smooth curve. Assume that the fiber $\mathcal{X}_{t}$ is smooth for $t \neq 0$, and has at worst ordinary quadratic singularities for $t=0$. Then

(i) If for general $t \in B, \mathcal{X}_{t}$ admits a Chow theoretic decomposition of the diagonal (equivalently, $\mathrm{CH}_{0}\left(\mathcal{X}_{t}\right)$ is universally trivial), the same is true for any smooth projective model $\widetilde{\mathcal{X}}_{o}$ of $\mathcal{X}_{o}$.

(ii) If for general $t \in B, \mathcal{X}_{t}$ admits a cohomological decomposition of the diagonal, and the even degree integral homology of a smooth projective model $\widetilde{\mathcal{X}}_{o}$ of $\mathcal{X}_{o}$ is algebraic (i.e. generated over $\mathbb{Z}$ by classes of subvarieties), $\widetilde{\mathcal{X}}_{o}$ also admits a cohomological decomposition of the diagonal.

SKETCH OF PROOF. One first shows by a specialization argument (and this step does not need any assumption on the singularities of the fibers) that under the assumptions made on the general fibers, the central fiber admits a Chow decomposition (case (i)) or a homological decomposition of the diagonal (case (ii)). Note that we have the notion of a homological decomposition of the diagonal even for singular projective varieties: we just need to know that cycles have a homology class, which is standard.

The second step is passing from $\mathcal{X}_{o}$ to $\widetilde{\mathcal{X}}_{o}$ and this is here that we use the assumption on the singularities. Let us concentrate on (i). From the decomposition

$$
\Delta \mathcal{X}_{o}=\mathcal{X}_{o} \times x+Z \text { in } \mathrm{CH}_{n}\left(\mathcal{X}_{o} \times \mathcal{X}_{o}\right)
$$


where $Z$ is supported on $D \times \mathcal{X}_{o}$, we deduce by restriction to

$$
U \times U, \quad U:=\mathcal{X}_{o} \backslash \operatorname{Sing} \mathcal{X}_{o}=\widetilde{\mathcal{X}}_{o} \backslash E,
$$

where $E$ is the exceptional divisor of the resolution singularities of $\mathcal{X}_{o}$ obtained by blowing-up the singular points:

$$
\Delta_{U}=U \times x+Z_{\mid U \times U} \text { in } \mathrm{CH}_{n}(U \times U) .
$$

By the localization exact sequence (1), we get a decomposition on $\widetilde{\mathcal{X}}_{o}$ which takes the following form:

$$
\Delta_{\tilde{\mathcal{X}}_{o}}=\widetilde{\mathcal{X}}_{o} \times x+\widetilde{Z}+\Gamma_{1}+\Gamma_{2} \text { in } \mathrm{CH}_{n}\left(\widetilde{\mathcal{X}}_{o} \times \widetilde{\mathcal{X}}_{o}\right),
$$

where $\widetilde{Z}$ is supported on $D^{\prime} \times \widetilde{\mathcal{X}}_{o}$ for some $D^{\prime} \varsubsetneqq \widetilde{\mathcal{X}}_{o}$, and $\Gamma_{1}$ is supported on $E \times \widetilde{\mathcal{X}}_{o}, \Gamma_{2}$ is supported on $\widetilde{\mathcal{X}}_{o} \times E$. Of course the cycle $\Gamma_{1}$ does not dominate $\widetilde{\mathcal{X}}_{o}$ by the first projection, so we need only to understand $\Gamma_{2}$. But $E$ is a disjoint union of smooth quadrics $Q_{i}$ of dimension $\geq 1$, and for each of them, $n$-dimensional cycles in $\widetilde{\mathcal{X}}_{o} \times Q_{i}$ decompose as $\alpha_{i} \widetilde{\mathcal{X}}_{o} \times x_{i}+Z_{i}$, where $Z_{i}$ does not dominate $\widetilde{\mathcal{X}}_{o}$ by the first projection. This concludes the proof if one realizes that the $x_{i}$ 's are all rationally equivalent in $\widetilde{\mathcal{X}}_{o}$ (this is where we use $n \geq 2$ ).

Theorem 4.13, (i), has been improved in [12] which proves the same result under weaker assumptions on the singularities. This has been used in $[\mathbf{1 2}],[\mathbf{7}],[\mathbf{1 9}],[\mathbf{3 5}]$ to get many new applications. We will describe below only our original application of the method.

4.3.2. The very general double solid is not stably rational. Recall that a quartic double solid is a hypersurface $X$ in $\mathbb{L}:=\operatorname{Spec}\left(\operatorname{Sym} \mathcal{O}_{\mathbb{P}^{3}}(-2)\right)$ defined by the equation $u^{2}=p^{*} f$, where $u$ is the canonical extra section of $\pi^{*} \mathcal{O}_{\mathbb{P}^{3}}(2)$ on $\mathbb{L}$ and $f \in H^{0}\left(\mathbb{P}^{3}, \mathcal{O}_{\mathbb{P}^{3}}(4)\right)$. Thus quartic double solids are parameterized by $\mathbb{P}\left(H^{0}\left(\mathbb{P}^{3}, \mathcal{O}_{\mathbb{P}^{3}}(4)\right)\right)$. We described in Section 2.1.3 the Artin-Mumford double solid $X_{o}$ which is nodal, with the property that $\widetilde{X}_{o}$ has a nontrivial Artin-Mumford invariant.

THEOREM 4.14. The very general double solid $X$ does not admit a cohomological (hence a fortiori Chow-theoretic) decomposition of the diagonal. Similarly, the desingularization of the very general double solid $X$ with $k \leq 7$ nodes in general position does not admit a cohomological decomposition of the diagonal.

Here we observe that given $k \leq 7$ general points in $\mathbb{P}^{3}$, there is a linear space of dimension $34-4 k$ of quartic homogeneous polynomials having 0 differential at these 7 points. There is thus an irreducible variety parameterizing quartic double solids with $k$ nodes in general position. As usual, very general means that the statement is true for an equation $f$ in the complement of a countable union of proper closed algebraic subsets of this variety. 
Theorem 4.14 immediately follows from Theorem 4.13 by degeneration to the Artin-Mumford double solid. Indeed, if $X_{o}$ is the Artin-Mumford double solid, $\widetilde{X}_{o}$ does not admit a cohomological decomposition of the diagonal by Proposition 4.9. For the nodal case, one needs to check that the ArtinMumford double solid smoothifies partially to the $k$-nodal quartic double solid with $k$ nodes in general position, for $k \leq 7$.

A a consequence of Theorem 4.14, one gets the following

THEOREM 4.15. The desingularization of the very general double solid with $k \leq 7$ nodes in general position is not stably rational.

Note that by Endrass [14], if $\widetilde{X}$ is as in Theorem 4.15, $\widetilde{X}$ has trivial Artin-Mumford invariant. In fact Endrass proves that the desingularization of a quartic double solid with less than 10 points has no torsion in its third Betti cohomology. To our knowledge, the only criterion for stable irrationality of rationally connected threefolds used previously was the Artin-Mumford invariant.

4.3.3. Further results. Theorem 4.14 has for consequence the following result:

THEOREM 4.16. Let $X$ be the desingularization of the very general double solid with 7 nodes in general position. Then there is no universal codimension 2 cycle on $J^{3}(X) \times X$.

Proof. Indeed, we know by Theorem 4.14 that $X$ has no cohomological decomposition of the diagonal. On the other hand, by $[\mathbf{1 4}]$, Tors $H_{B}^{3}(X, \mathbb{Z})=$ 0 and as $X$ is rationally connected this implies that Tors $H_{B}^{*}(X, \mathbb{Z})=0$. Also $Z^{4}(X)=0$ by [39] since $X$ is rationally connected. Finally, we have $\operatorname{dim} J^{3}(X)=3$, hence $\left(J^{3}(X), \Theta_{X}\right)$ is a Jacobian in this case, so that the minimal class $\theta^{2} / 2$ is algebraic on $J^{3}(X)$. Theorem 4.12 then tells us that $X$ does not have a universal codimension 2 cycle.

We conclude with a sketch of the results obtained in $[\mathbf{4 2}]$ concerning cubic hypersurfaces. There are two challenging open problems concerning cubic hypersurfaces, namely the conjectured irrationality of the very general cubic fourfold and the question whether a cubic threefold is stably rational or not. We do not have definite results on either of these questions, but we studied in [42] decomposition of the diagonal (or universal $\mathrm{CH}_{0}$ group) for smooth cubic hypersurfaces. Our main result is the following:

TheOREM 4.17. [42, Theorem 1.1] Let $X$ be a smooth cubic hypersurface such that the group $H_{B}^{2 *}(X, \mathbb{Z}) / H_{B}^{2 *}(X, \mathbb{Z})_{\text {alg }}$ has no 2-torsion. (This includes odd dimensional cubics and cubic fourfolds.) Then $X$ has a Chow decomposition of the diagonal (that is $\mathrm{CH}_{0}(X)$ is universally trivial) if and only if $X$ admits a cohomological decomposition of the diagonal.

As it is much easier to study cohomological decompositions of the diagonal, we get various consequences. 
THEOREM 4.18. [42, Theorem 5.6] Let $X$ be a special cubic fourfold in the sense of Hassett [18], whose discriminant is not divisible by 4. Then $\mathrm{CH}_{0}(X)$ is universally trivial.

Concerning cubic threefolds, we conclude using Theorem 4.12 that we have the following equivalence

Theorem 4.19. [42, Theorem 1.7] A smooth cubic threefold $X$ has universally trivial $\mathrm{CH}_{0}$ group if and only if the minimal class $\theta^{4} / 4$ ! is algebraic on $J^{3}(X)$.

To state the last result, let us introduce the following definition.

DeFinition 4.20. Let $X$ be an algebraic variety over a field $K$ and let $Y \subset X$ be a closed algebraic subset. Then $\mathrm{CH}_{0}(Y) \rightarrow \mathrm{CH}_{0}(X)$ is universally surjective if for any field $L$ containing $K, \mathrm{CH}_{0}\left(Y_{L}\right) \rightarrow \mathrm{CH}_{0}\left(X_{L}\right)$ is surjective.

Applying the definition to $L=K(X)$ we see immediately that this is equivalent to the existence of a decomposition of the diagonal

$$
\Delta_{X}=Z_{1}+Z_{2} \text { in } \mathrm{CH}^{n}(X \times X),
$$

where $Z_{1}$ is supported on $D \times X$ for some $D \varsubsetneqq X$, and $Z_{2}$ supported on $X \times Y$. We will say that the essential $\mathrm{CH}_{0}$ dimension of $X$ is $k$ if the minimal dimension of a closed algebraic subset $Y \subset X$ with $\mathrm{CH}_{0}(Y) \rightarrow \mathrm{CH}_{0}(X)$ universally surjective is $k$.

Theorem 4.21. [42, Theorem 1.3] Let $X$ be a very general cubic hypersurface of dimension $n$. Then the essential $\mathrm{CH}_{0}$ dimension of $X$ is either 0 or $n$.

\section{References}

[1] E. Arbarello, M. Cornalba,Ph. Griffiths, J. Harris. Geometry of algebraic curves, Vol. I, Grundlehren der Mathematischen Wissenschaften 267. Springer-Verlag, New York (1985).

[2] M. Artin, D. Mumford. Some elementary examples of unirational varieties which are not rational, Proc. London Math. Soc. (3) 25 (1972), 75-95.

[3] M. Atiyah et F. Hirzebruch, Analytic cycles on complex manifolds, Topology 1, 25-45 (1962).

[4] A. Auel, J.-L. Colliot-Thélène, R. Parimala. Universal unramified cohomology of cubic fourfolds containing a plane, preprint arXiv:1310.6705.

[5] A. Beauville. Surfaces algébriques complexes, Astérisque 54, SMF (1978).

[6] A. Beauville. Variétés de Prym et jacobiennes intermédiaires. Ann. Sci. Éc. Norm. Sup. 10, 309-391 (1977).

[7] A. Beauville. A very general sextic double solid is not stably rational, Bull. Lond. Math. Soc. 48 (2016), no. 2, 321-324.

[8] S. Bloch, V. Srinivas. Remarks on correspondences and algebraic cycles, Amer. J. of Math. 105 (1983) 1235-1253.

[9] I. Cheltsov. Birationally rigid Fano varieties. (Russian) Uspekhi Mat. Nauk 60 (2005), no. 5(365), 71-160; translation in Russian Math. Surveys 60 (2005), no. 5, 875-965. 
[10] H. Clemens, Ph. Griffiths. The intermediate Jacobian of the cubic threefold, Annals of Mathematics. Second Series 95 (2): 281-356 (1972).

[11] J.-L. Colliot-Thélène, M. Ojanguren. Variétés unirationnelles non rationnelles: audelà de l'exemple d'Artin et Mumford, Invent. Math. 97 (1989), no. 1, 141-158.

[12] J.-L. Colliot-Thélène, A. Pirutka. Hypersurfaces quartiques de dimension 3: nonrationalité stable, Annales scientifiques de l'ENS 49, fascicule 2 (2016), 371-397.

[13] J.-L. Colliot-Thélène, C. Voisin. Cohomologie non ramifiée et conjecture de Hodge entière, Duke Math. Journal, Volume 161, Number 5, 735-801 (2012).

[14] S. Endrass. On the divisor class group of double solids, Manuscripta Math. 99, 341-358 (1999).

[15] W. Fulton.Intersection theory, Ergebnisse der Mathematik und ihrer Grenzgebiete 2. Springer-Verlag, Berlin, (1984).

[16] T. Graber, J. Harris, J. Starr. Families of rationally connected varieties. J. Amer. Math. Soc., 16(1), 57-67 (2003).

[17] Ph. Griffiths. On the periods of certain rational integrals. I, II. Ann. of Math. (2) 90 (1969), 460-495; ibid. (2) 90 (1969) 496-541.

[18] B. Hassett. Special cubic fourfolds. Compositio Math. 120 (2000), no. 1, 1-23.

[19] B. Hasset, A. Kresch, Yu. Tschinkel. Stable rationality and conic bundles , arXiv:1503.08497, to appear in Math. Annalen.

[20] A. Höring, C. Voisin. Anticanonical divisors and curve classes on Fano manifolds, Pure and Applied Mathematics Quarterly, Volume 7, Number 4 (Special Issue: In memory of Eckart Viehweg), 1371-1393 (2011).

[21] V. A Iskovskikh. On the rationality problem for conic bundles. Duke Math. J. 54 (1987), no. 2, 271-294.

[22] V. A Iskovskikh, Yu. Manin. Three-dimensional quartics and counterexamples to the Lüroth problem. Mat. Sb. (N.S.) 86(128) (1971), 140-166.

[23] J. Kollár. Lemma p. 134 in Classification of irregular varieties, edited by E. Ballico, F. Catanese, C. Ciliberto, Lecture Notes in Math. 1515, Springer

[24] J. Kollár. Nonrational hypersurfaces. J. Amer. Math. Soc. 8 (1995), 241-249 (1990).

[25] A. Kuznetsov. Derived categories of cubic fourfolds, in Cohomological and geometric approaches to rationality problems, 219-243, Progr. Math., 282, Birkhäuser Boston, Inc., Boston, MA, (2010).

[26] D. Markushevich and A. Tikhomirov. The Abel-Jacobi map of a moduli component of vector bundles on the cubic threefold, J. Algebraic Geometry 10 (2001), 37-62.

[27] T. Matsusaka. On a characterization of a Jacobian variety. Memo. Coll. Sci. Univ. Kyoto. Ser. A. Math. 32 (1959) 1-19.

[28] D. Mumford. Rational equivalence of 0-cycles on surfaces. J. Math. Kyoto Univ. 9, 195-204 (1968).

[29] J. P. Murre. Applications of algebraic K-theory to the theory of algebraic cycles, in Proc. Conf. Algebraic Geometry, Sitjes 1983, LNM 1124 (1985), 216-261, SpringerVerlag.

[30] E. Peyre. Unramified cohomology and rationality problems, Math Ann. 296 (1993), 247-268.

[31] J.-P. Serre. On the fundamental group of a unirational variety. J. London Math. Soc. 34 (1959) 481-484.

[32] J.-P. Serre. Géométrie algébrique et géométrie analytique. Ann. Inst. Fourier, Grenoble 6 (1955-1956), 1-42.

[33] Ch. Soulé, C. Voisin. Torsion cohomology classes and algebraic cycles on complex projective manifolds, Advances in Mathematics, Vol 198/1 pp 107-127 (2005).

[34] Z. Tian, R. Zong. One-cycles on rationally connected varieties. Compos. Math. 150 (2014), no. 3, 396-408.

[35] B. Totaro. Hypersurfaces that are not stably rational. J. Amer. Math. Soc. 29 (2016), no. $3,883-891$. 
[36] C. Voisin. Unirational threefolds with no universal codimension 2 cycle, Invent. Math. 201 (2015), no. 1, 207-237.

[37] C. Voisin. Remarks on curve classes on rationally connected varieties, Clay Mathematics Proceedings Volume 18, 591-599 (2013).

[38] C. Voisin. Abel-Jacobi map, integral Hodge classes and decomposition of the diagonal, J. Algebraic Geom. 22 (2013), no. 1, 141-174.

[39] C. Voisin. On integral Hodge classes on uniruled and Calabi-Yau threefolds, in Moduli Spaces and Arithmetic Geometry, Advanced Studies in Pure Mathematics 45, 2006, pp. 43-73.

[40] C. Voisin. Some aspects of the Hodge conjecture, Japan. J. Math. 2, 261-296 (2007).

[41] C. Voisin. Degree 4 unramified cohomology with finite coefficients and torsion codimension 3 cycles, in Geometry and Arithmetic, (C. Faber, G. Farkas, R. de Jong Eds), Series of Congress Reports, EMS 2012, 347-368.

[42] C. Voisin. On the universal $C H_{0}$ group of cubic hypersurfaces, arXiv:1407.7261 to appear in JEMS.

[43] C. Voisin. Hodge theory and Complex Algebraic Geometry I, Cambridge University Press (2002).

[44] C. Voisin. Hodge theory and Complex Algebraic Geometry II, Cambridge University Press(2003).

CNRS, Institut de Mathématiques de Jussieu, 4 Place Jussieu, 75252 Paris CEDEX 05, France

E-mail address: claire.voisin@imj-prg.fr 